\title{
Efficacy and safety of iron-chelation therapy with deferoxamine, deferiprone, and deferasirox for the treatment of iron-loaded patients with non- transfusion-dependent thalassemia syndromes
}

\author{
This article was published in the following Dove Press journal: \\ Drug Design, Development and Therapy \\ 29 January 2016 \\ Number of times this article has been viewed
}

\section{Christina N Kontoghiorghe \\ George J Kontoghiorghes}

Postgraduate Research Institute of Science, Technology, Environment and Medicine, Limassol, Cyprus
Correspondence: George J Kontoghiorghes Postgraduate Research Institute of Science, Technology, Environment and Medicine, 3 Ammochostou Street, Limassol 3021, Cyprus

Tel +357 26272076

Fax +357 2627 I 434

Email kontoghiorghes.g.j@pri.ac.cy
Abstract: The prevalence rate of thalassemia, which is endemic in Southeast Asia, the Middle East, and the Mediterranean, exceeds 100,000 live births per year. There are many genetic variants in thalassemia with different pathological severity, ranging from a mild and asymptomatic anemia to life-threatening clinical effects, requiring lifelong treatment, such as regular transfusions in thalassemia major (TM). Some of the thalassemias are non-transfusion-dependent, including many thalassemia intermedia (TI) variants, where iron overload is caused by chronic increase in iron absorption due to ineffective erythropoiesis. Many TI patients receive occasional transfusions. The rate of iron overloading in TI is much slower in comparison to TM patients. Iron toxicity in TI is usually manifested by the age of 30-40 years, and in TM by the age of 10 years. Subcutaneous deferoxamine (DFO), oral deferiprone $\left(\mathrm{L}_{1}\right)$, and DFO- $\mathrm{L}_{1}$ combinations have been effectively used for more than 20 years for the treatment of iron overload in TM and TI patients, causing a significant reduction in morbidity and mortality. Selected protocols using DFO, $\mathrm{L}_{1}$, and their combination can be designed for personalized chelation therapy in TI, which can effectively and safely remove all the excess toxic iron and prevent cardiac, liver, and other organ damage. Both $\mathrm{L}_{1}$ and DF could also prevent iron absorption. The new oral chelator deferasirox (DFX) increases iron excretion and decreases liver iron in TM and TI. There are drawbacks in the use of DFX in TI, such as limitations related to dose, toxicity, and cost, iron load of the patients, and ineffective removal of excess iron from the heart. Furthermore, DFX appears to increase iron and other toxic metal absorption. Future treatments of TI and related iron-loading conditions could involve the use of the iron-chelating drugs and other drug combinations not only for increasing iron excretion but also for preventing iron absorption.

Keywords: thalassemia intermedia, chelation therapy, deferoxamine, deferiprone, deferasirox, iron overload, iron absorption, efficacy, safety

\section{Introduction}

Iron is an essential metal found in all living organisms, and is involved in many physiological processes, such as oxygen transport and energy transduction. ${ }^{1,2}$ It is estimated that more than a quarter of the human population is affected at some stage of their life by abnormalities of iron metabolism, in particular iron-deficiency anemia. Similarly, many millions of people suffer from iron overload, including hereditary hemochromatosis and thalassemia intermedia (TI), which are caused by increased iron absorption..$^{3-6}$ In these and other iron-loading conditions, excess iron is toxic, causing increased morbidity and mortality as a result of tissue damage. Iron plays an important catalytic role in free radical pathology and oxidative stress damage, which is not only observed in iron-overload 
diseases but also in non-iron-loaded diseases of focal or labile iron toxicity, such as cardiovascular, neurodegenerative, hepatic, and renal diseases, as well as in cancer and aging. ${ }^{7,8}$

A major group of diseases related to iron abnormalities involving millions of people are the inherited hemoglobinopathies, especially thalassemia and sickle-cell disease. It is estimated that the prevalence of these two inherited hemoglobinopathies exceeds 200,000 live births per year, with approximately 100,000 for each disease. ${ }^{3,4}$ Sickle-cell disease is endemic in Africa, Central and South America, and the Afro-American population of North America, and is also distributed with lower frequency in Middle East and the Mediterranean countries. Thalassemia is endemic mainly in Southeast Asia, the Middle East, and Mediterranean countries.

There are many genetic variants of thalassemia related to the hemoglobin $(\mathrm{Hb})$ chains $(\alpha, \beta, \gamma, \delta$, and $\varepsilon)$. Many of the thalassemia variants can cause pathological effects of different severity, ranging from a mild and asymptomatic anemia to death, eg, in hydrops fetalis in $\alpha$-thalassemia (4/4 $\alpha$-globin gene deletion). ${ }^{9}$ A major group of thalassemia patients with life-threatening clinical effects requiring life long treatments, such as regular red blood cell transfusions within a few years of life, is $\beta$ thalassemia major (TM) $(2 / 2$ $\beta$-globin gene deletion). ${ }^{3,4}$ Iron overload in TM is caused mainly from chronic transfusions and to a lesser extent by increased iron absorption. Another major group of thalassemias of milder severity than TM are the iron-loaded nontransfusion-dependent thalassemias (IL-NTDTs), which include many TI variants where iron overload is caused by chronic increase in iron absorption due to ineffective erythropoiesis. ${ }^{3,4,9-12}$ The same mechanism, but with a higher rate of iron absorption, is also observed in nontransfused TM patients in developing countries, where no transfusion or chelation therapy is available.

Many patients with IL-NTDT may have a number of red blood-cell transfusions because of low Hb levels, infections, and other causes. The rate of transfusion increases with age in most cases of IL-NTDT, eg, many TI patients at the age of 30-50 years may be regularly transfused at a rate similar to that of TM patients..$^{9-12}$ The rate of iron overload from increased iron absorption in IL-NTDT is generally much slower in comparison to iron accumulated from transfusions in regularly transfused TM patients. ${ }^{12,13}$ However, the rate of iron absorption in IL-NTDT is generally similar to that observed in hereditary hemochromatosis, and in both conditions iron overload is manifested in many cases very early in life, even from early childhood..$^{9-15}$

Genetic, dietary, immunological, and other factors influence the rate of iron-overload and related toxicity in both IL-NTDT and TM. Iron-overload toxicity is manifested in many organs, including the heart, liver, spleen, and pancreas. ${ }^{11,16-19}$ Iron overload in IL-NTDT can lead progressively to cardiomyopathy, liver fibrosis, cirrhosis, hepatocellular carcinoma, and diabetes. ${ }^{11,16-21}$ In addition to iron overload-related toxicity, ineffective erythropoiesis, and chronic anemia, there are also many other abnormalities related to the underlying condition in TI and other similar IL-NTDTs, such as hypothyroidism, hypogonadism, hypersplenism, pulmonary hypertension, and osteoporosis. ${ }^{11,16-21}$

Different clinical strategies are generally used for the treatment of TI and other similar IL-NTDT patients, which mainly include red blood-cell transfusions, increase in $\mathrm{HbF}$ production using hydroxycarbamide (hydroxyurea), splenectomy, and iron-chelation therapy. ${ }^{9-11}$

Iron-chelation therapy can decrease and in many cases eliminate the morbidity and mortality rates associated with iron toxicity in IL-NTDT syndromes. Iron-chelation strategies in patients with this syndrome are similar to those in TM, and are generally based on the early diagnosis and prevention of iron overload and toxicity and also the rapid normalization of the iron stores. ${ }^{14,15}$ The chelating drugs and chelation protocols used in IL-NTDT are similar to those in TM. Most of these protocols involve the administration of subcutaneous deferoxamine (DFO), oral deferiprone $\left(\mathrm{L}_{1}\right)$, and $\mathrm{DFO}-\mathrm{L}_{1}$ combinations, which have been effectively used for more than 20 years for the treatment of iron overload in TM and TI patients (Figure 1). ${ }^{14,15}$ In addition, the recent introduction of oral deferasirox (DFX) has increased the options for the treatment of iron overload in TM and other conditions, including IL-NTDT (Figure 1). Several studies sponsored by the manufacturers of DFX have been recently published on the effect of the drug on IL-NTDT. ${ }^{22}$

Personalized medicine is designed and appropriately used in each case for optimizing chelation therapy in patients with IL-NTDT and TM. This approach is generally based on the risk-benefit assessment of each chelator and chelation protocol, and also the idiosyncratic clinical profile of the patients, as determined by drug absorption, distribution, metabolism, excretion, and toxicity effects and other relevant parameters, such as organ function. ${ }^{23}$ However, other factors are also considered in the selection criteria of chelating drugs, including costs, influence of the pharmaceutical companies, and drug policies of the regulatory authorities. ${ }^{24,25}$

\section{Epidemiology and severity of iron- loaded non-transfusion-dependent thalassemia syndromes}

IL-NTDT is a syndrome characterized by excess iron deposition in organs similar to hereditary hemochromatosis, 
<smiles>Cc1c(O)c(=O)ccn1C</smiles>

Deferiprone<smiles>CC(=O)N(O)CCCCCNC(=O)CCC(=O)N(O)CCCCCNC(=O)CCC(=O)N(O)CCCCCN</smiles><smiles>NC(=O)NO</smiles>

Hydroxycarbamide

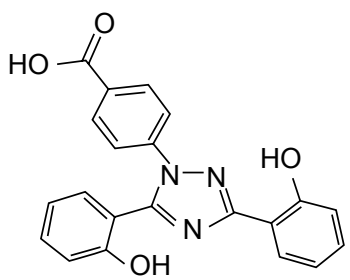

Deferasirox<smiles>Cc1occc(=O)c1O</smiles>

Maltol<smiles>Oc1cccc2cccnc12</smiles>

8-Hydroxyquinoline

Figure I Structure of the chelating drugs.

Notes: The chemical and physicochemical properties of chelating drugs influence their clinical activity, including their mode of action, organ targeting, efficacy, and toxicity. Deferiprone and deferoxamine are hydrophilic chelators that increase iron excretion and decrease iron absorption. Maltol, deferasirox, and 8-hydroxyquinoline are lipophilic chelators that form lipophilic metal complexes, and can cause an increase in iron and other metal absorption. Orally absorbed, nonmetal-bound deferasirox mobilizes excess iron, mainly from the liver, and causes an increase in iron excretion. The pharmacological effects of different drugs, eg, hydroxycarbamide (hydroxyurea) are affected by iron binding.

which is caused by increased body-iron intake as a result of an increase in net body-iron intake from gastrointestinal absorption in comparison to total body-iron excretion and other losses. ${ }^{12-15,26,27}$ The level of iron overload and its severity in this syndrome is related primarily to hereditary abnormalities, but also a number of metabolic, environmental, dietary, aging, and other factors influencing the ferrokinetics of iron metabolism through different mechanisms. ${ }^{12-15,26,27}$

In relation to the hereditary factors related to IL-NTDT syndromes, $\beta$-TI is the best-characterized genetic disorder, which is associated with a variety of gene mutations involving the $\beta$-globin chain of $\mathrm{Hb}$ and is predominantly found in Southeast Asia, Mediterranean, and Middle Eastern countries, similar to that of TM patients (Table 1). ${ }^{3,4}$ In TI patients, the normal production of $\mathrm{Hb}$ is affected to a variable extent depending on the genotype and age of the patient. Thousands of $\mathrm{TI}$ patients diagnosed with $\mathrm{Hb}$ variants of the $\alpha$-, $\beta$-, $\gamma, \delta$-, and $\varepsilon$-globin genes exhibit milder forms of anemia than TM patients (Table 1). ${ }^{3,4}$

There are several categories of TI patients where red blood-cell transfusions may be initiated to compensate for the decreasing level of $\mathrm{Hb}$ production. In some cases, the rate of transfusions may increase to levels similar to those observed in TM patients. There are also other groups of TI patients who may receive less frequent transfusions and some where transfusions are not necessary. In the latter group,
TI patients usually maintain $\mathrm{Hb}$ levels at $6-10 \mathrm{~g} / \mathrm{dL}$, which in many cases is sufficient in younger patients to be maintained without complications, and transfusion can be avoided. ${ }^{3,4}$ For example, increased production of $\mathrm{HbF}$ is associated with milder TI disease. In general, Hb production in TI decreases with age, and the general health of TI patients also deteriorates as they grow older..$^{10,11,15}$

Similar variations are observed in $\mathrm{HbE} \beta$-thalassemia patients, most of whom live in developing countries of Southeast Asia, and also in immigrants from this region. ${ }^{3,417,27,28}$ It is estimated that the $\mathrm{HbE} \beta$-thalassemia genotype is responsible for approximately $50 \%$ of all severe $\beta$-thalassemias worldwide (Table 1). The disorder is characterized by marked clinical variability and $\mathrm{Hb}$ production, ranging from a mild and asymptomatic anemia to a life-threatening disorder requiring transfusions from infancy. For example, no treatment is required for mild $\mathrm{HbE} \beta$-thalassemia that is characterized by normal $\mathrm{Hb}$ levels (9-12 g/dL). Patients in this category do not usually develop clinically significant symptoms. However, more severe forms are characterized by decreased $\mathrm{Hb}$ levels $(6-8 \mathrm{~g} / \mathrm{dL})$ and the clinical manifestations are similar to those of TI, where transfusions are not required unless infections or other complications increase the anemia further. Most patients of $\mathrm{HbE} \beta$-thalassemia ( $\sim 50 \%)$ are thought to be included in this category of IL-NTDT, where iron overload from progressive 
Table I Examples of thalassemia intermedia and other conditions where iron overload can be caused from the increased gastrointestinal absorption of iron*

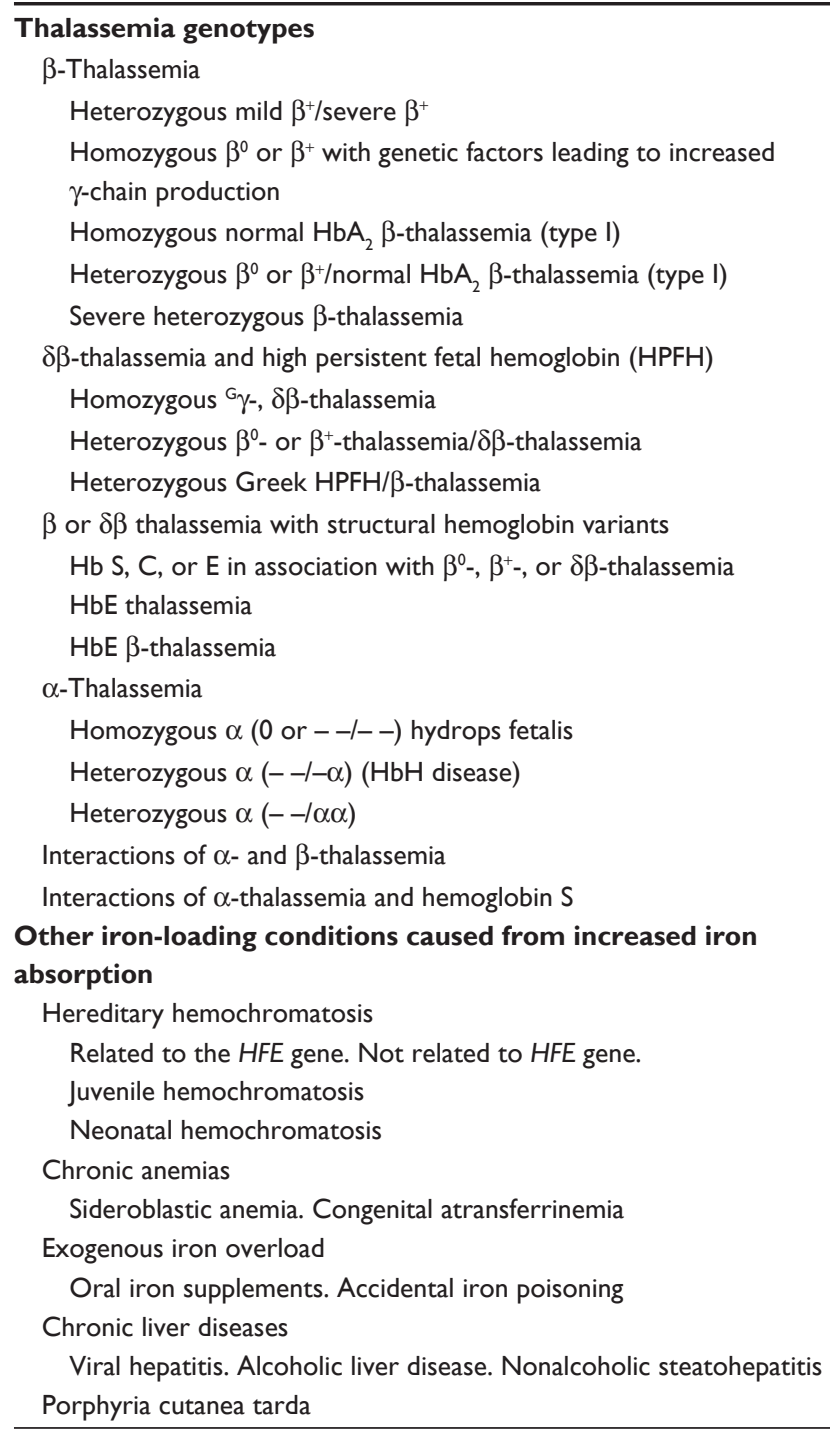

Notes: *Increased gastrointestinal iron absorption is observed in different thalassemia intermedia genotypes, hereditary hemochromatosis, and other conditions, which can lead to iron overload. The main diagnostic criteria for iron overload are increased serum ferritin and transferrin iron-saturation levels and decrease in the signal intensity of magnetic resonance imaging $\mathrm{T}_{2}$ or $\mathrm{T}_{2}^{*}$ in the liver and heart, due to excess iron accumulation and deposition.

Abbreviation: $\mathrm{Hb}$, hemoglobin.

increases in iron absorption may occur. It is estimated that about a third of the cases of $\mathrm{HbE} \beta$-thalassemia are present with the severe form of the disease, which is characterized by very low $\mathrm{Hb}$ levels $(4-5 \mathrm{~g} / \mathrm{dL})$, and the clinical symptoms are similar to those of TM patients. Regular red blood-cell transfusions and iron-chelation therapy are required for the survival of this category of $\mathrm{HbE} \beta$-thalassemia patients similar to TM patients. ${ }^{3,4,17,27,28}$

There are many other categories of patients with similar rates of body-iron loading to that of IL-NTDT that are due to increased iron absorption and require chelation therapy (Table 1). These include hereditary hemochromatosis patients who cannot be treated with venesection because of complications, such as severe congestive heart failure, cardiomyopathy, severe anemia, advanced liver cirrhosis, or poor peripheral venous access, and also patients with porphyria cutanea tarda (Table 1). ${ }^{15}$ Additionally, there are other categories of patients, such as those with sickle-cell anemia or sickle-cell anemia with $\beta$-thalassemia, with similar rates of body-iron loading to that of IL-NTDT, which are caused by occasional red blood-cell transfusions (Table 1). ${ }^{10,11,15}$ In all these cases, the body-iron intake from increased gastrointestinal absorption and occasional red blood-cell transfusions is much lower and iron accumulation is at a slower rate in comparison to regularly transfused TM patients. Accordingly, the toxic side effects of iron overload are manifested at different ages in IL-NTDT, and less intensive chelation therapy may be used at younger ages for the treatment of iron overload and toxicity in comparison to TM.

\section{Molecular mechanisms of increased iron absorption and storage in iron- loaded non-transfusion-dependent thalassemias}

Under normal conditions, the regulatory uptake and utilization of iron appear to involve a number of pathways and proteins that control the rate of iron transfer from the gastrointestinal tract to the bloodstream such as ferrireductase(s), DMT1, ferroportin, hepcidin, transferrin, and ferritin (Figure 2) ${ }^{1,2}$ Different changes in the regulatory mechanisms of dietary iron uptake from the gastrointestinal tract are suggested for causing increased iron absorption in hereditary hemochromatosis, TI, and other similar conditions. ${ }^{1,2,15}$

The mechanism and the regulatory pathway of iron absorption is thought to involve initially the conversion of dietary ferric iron to ferrous iron by a ferrireductase present at the cell surface of the enterocyte and then its intracellular transport by DMT1 (Figure 2). Once inside the cell, iron is partly incorporated into ferritin or transferred into the lowmolecular-weight intracellular iron pool. Some of the iron is then transported to ferroportin at the basolateral membrane of the enterocyte, which exports iron into the plasma. The critical role of iron absorption is thought to be played by hepcidin, a peptide hormone produced in the liver. The mode of action of hepcidin is the prevention of iron release into plasma by binding ferroportin, causing its internalization and degradation within the enterocyte. Following the binding of ferroportin by hepcidin, iron is not released into plasma, 


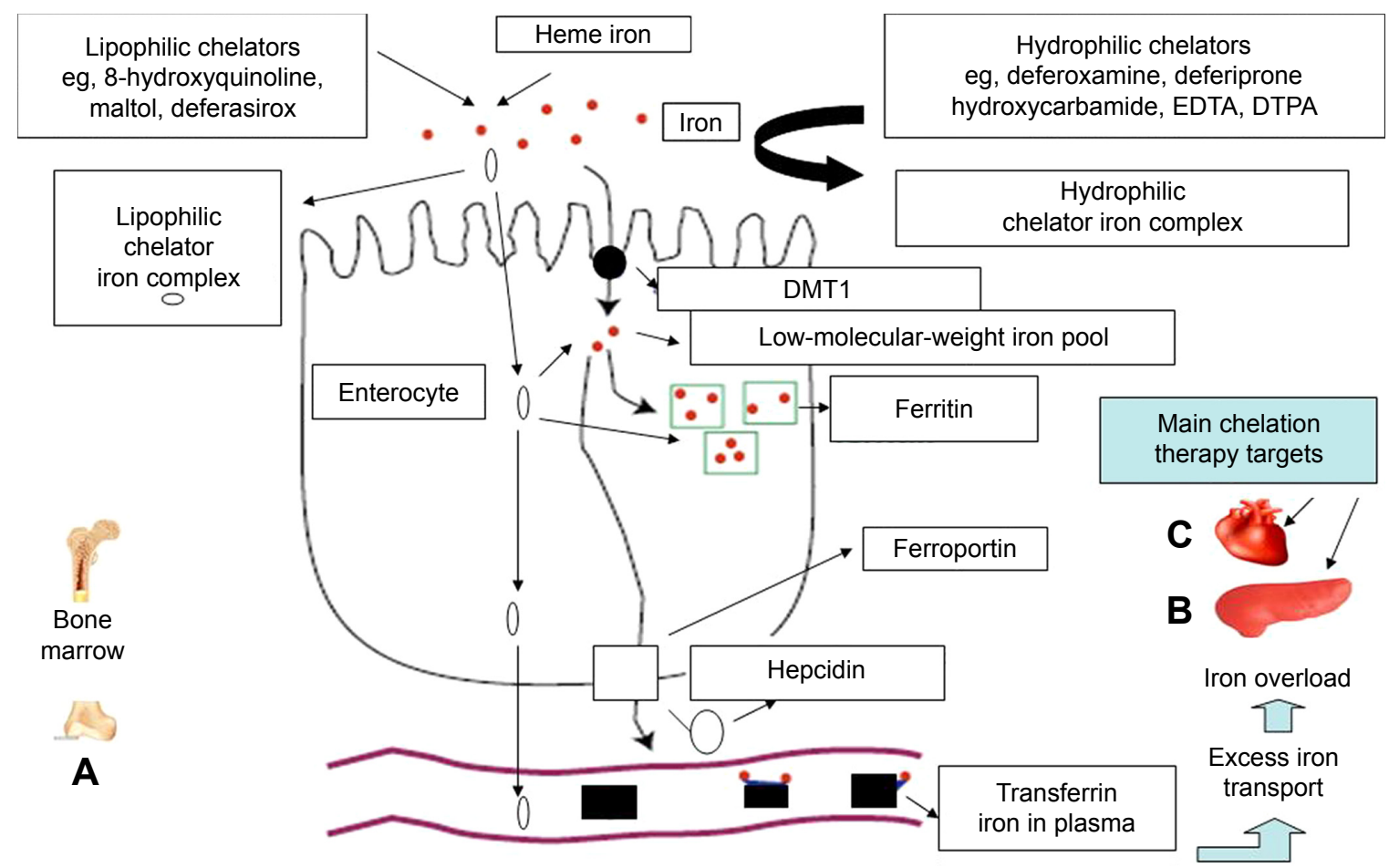

Figure 2 Iron-absorption and iron-overload mechanisms in non-transfusion-dependent thalassemias: the role of chelators and chelating drugs.

Notes: Mechanism of iron absorption at the enterocyte using regulatory pathways of iron metabolism involving DMTI, hepcidin, ferroportin, and transferrin. Increased gastrointestinal iron absorption and iron overload is observed in non-transfusion-dependent thalassemias, due to ineffective erythropoiesis in the bone marrow (A). The level of increased iron absorption depends on the form and quantity of iron present in the diet and other factors, such as the presence of natural or synthetic iron chelators in the gastrointestinal tract. Iron-chelating drugs and other chelators have variable pharmacological effects on iron absorption, with lipophilic chelators causing an increase in iron absorption and hydrophilic chelators a decrease in iron absorption. Excess iron absorption causes iron overload and damage in the liver, the heart, and other organs. The liver is the main organ of excess iron deposition, whereas the heart is the most susceptible organ of iron toxicity, as a result of iron overload from increased iron absorption. Iron overload in both the liver (B) and the heart (C) in non-transfusion-dependent thalassemia are the main target sites of chelation therapy.

Abbreviations: DMTI, divalent metal transporter I; EDTA, ethylenediaminetetraacetic acid; DTPA, diethylenetriaminepentaacetic acid.

but remains within the enterocytes and returns in the gut lumen, since enterocytes are shed every $2-3$ days. Within this context, a decrease in plasma hepcidin concentration will increase iron absorption. ${ }^{1,2,15}$

Iron released into plasma from the basolateral membrane of the enterocyte by ferroportin is mobilized by plasma transferrin and transferred to all the cells of the body. The uptake of transferrin iron by cells is mediated by transferrin receptors that are present at the cell surface. Increased transferrin receptors are present especially in hepatocytes for iron storage and the erythroid cells for $\mathrm{Hb}$ synthesis. Intracellular release of iron is mediated by receptor-mediated endocytosis, through the internalization in a vesicle and acidification of the transferrin receptor-transferrin-iron complex. ${ }^{1,2}$

The expression of hepcidin, DMT1, and ferroportin appears to be affected by a number of factors. In particular, the signal to the intestine to increase iron absorption appears to be influenced by regulatory molecules (iron-responsive elements) which sense the iron stores. ${ }^{1,2,29,30}$ Different ironresponsive element regulatory molecules are also present, which appear to sense the production of $\mathrm{Hb}$ or anemia.
In both cases, the influence of the regulatory molecules cause an increase in the expression of DMT1 and a corresponding increase in the uptake of iron. In general, it would appear that in TI and other IL-NTDT, the signal arriving at the enterocytes is a combination of ineffective erythropoiesis, anemia, and hypoxia, which leads to the initiation of compensatory mechanisms, including an increase in plasma levels of erythropoietin, as well as a decrease in plasma levels of hepcidin. The decrease in hepcidin increases the rate of transfer of iron by ferroportin on the intestinal epithelium into the bloodstream, followed by an increase of transferrin iron uptake, transferrin iron delivery, and intracellular storage, mainly in the liver hepatocytes but also in other organs, including the heart and the pancreas. ${ }^{12,13,16-19}$

The level of iron overload in IL-NTDT patients is mainly characterized by increases in serum ferritin, plasma iron, and transferrin iron saturation, as well as by increases in iron concentration in the liver, heart, and other organs, which can be measured by magnetic resonance imaging (MRI) $\mathrm{T}_{2}$ and $\mathrm{T}_{2}$ * relaxation-time values. ${ }^{10-12,16,17,31,32}$ The same methods are also used for monitoring the progress of chelation therapy, 
as well as other treatments used for the reduction of iron absorption or the elimination of excess iron in organs of patients with IL-NTDT.

Iron overload has important clinical consequences in both patients with TI and TM. However, unlike TM, where iron overload is mostly caused from transfused red blood cells and is initially stored in the Kupffer cells of the liver, the iron overload caused in IL-NTDT originates from the absorption and intake of excess iron present in the gastrointestinal tract, which is mainly delivered and stored in the hepatocytes. Since iron accumulation primarily occurs in the hepatocytes, iron overload can predispose patients to liver fibrosis and cirrhosis, hepatocellular carcinoma, and other toxic side effects similar to hereditary hemochromatosis patients. ${ }^{20,21,30,31}$ Cardiac complications, including cardiac iron overload, are also very common in TI and other IL-NTDT patients, and in both cases appropriate therapeutic interventions are needed. ${ }^{16-20,30,31}$

\section{Nongenetic factors influencing the rate of iron absorption and body- iron intake in iron-loaded non- transfusion-dependent thalassemias}

The genotype of thalassemia is the major factor affecting the rate of gastrointestinal iron absorption and body-iron loading in IL-NTDT. However, there are many other additional factors that also appear to influence this process, and this is reflected by the wide variation in iron-loading in IL-NTDT patients with the same genotype. Some of these factors may be influenced by changes in relation to the iron-metabolism pathways and related proteins and regulatory molecules, such as hepcidin and ferroportin. Infection, inflammation, and pathological conditions may also influence normal iron metabolism and the rate of iron absorption in IL-NTDT (Table 2). ${ }^{1,2,5,6,10,11}$

Dietary habits are also important factors affecting the rate of iron absorption in IL-NTDT patients, but also in normal individuals. Within this context, the quantity and quality (ferrous, ferric, heme) of iron in food, as well as other dietary factors or constituents, such as ascorbic acid, polyphenols, inorganic phosphates, and tannins, can also influence the rate of iron absorption. ${ }^{15,26}$ Increased iron absorption is anticipated when dietary iron is in the form of heme, in comparison to ferrous iron and to a lesser extent ferric iron, which is the least soluble and absorbable form. ${ }^{15,26}$ The presence of reducing agents, such as ascorbic acid, could increase the absorption of iron by reducing ferric to ferrous iron. Low $\mathrm{pH}$ and dietary factors that increase the solubility of iron can also increase the
Table 2 Examples of nonregulatory factors affecting iron absorption*

Quantity of iron present in the diet, eg, vegetarian meals contain less iron

Quality of iron (ferrous, ferric, heme, ferritin, hemosiderin) in the diet Presence of dietary reducing agents, eg, ascorbic acid

Presence of dietary molecules with chelating properties, eg, tannins and polyphenols

Presence of oral drugs with chelating properties, eg, tetracycline and hydroxycarbamide

The quantity of water, alcohol, and other fluid intake in the gastrointestinal tract

Drugs and dietary molecules affecting iron transport across the enterocyte (eg, nifedipine, which is an L-type calcium-channel blocker)

Dietary factors affecting the solubilization of iron

$\mathrm{pH}$ of the stomach and intestine

Modulators of DMTI, hepcidin, ferroportin, and transferrin activity

Gastrectomy and other interventions where gastrointestinal function is affected

Infectious, inflammatory, chronic liver disease, and other diseases Aging, sports

Exogenous iron supplementation

Chronic iron supplementation. Oral iron supplements. Use of iron cooking utensils

Notes: *Many dietary, nonregulatory, and other factors such as those described in this table can affect the absorption of iron under normal conditions. The same and similar factors, as well as other regulatory factors, such as the erythropoietic activity of the bone marrow in thalassemia intermedia and other iron-loaded non-transfusiondependent thalassemias where ineffective erythropoiesis is observed, can also affect the rate of iron absorption and can lead to variable levels of iron deposition and overload in the liver, the heart, and other organs of the affected patients.

Abbreviation: DMTI, divalent metal transporter I.

absorption of iron (Table 2). ${ }^{15,26}$ In contrast, decrease in iron intake and iron deficiency is generally observed in vegetarians, where absorbable dietary iron is low in concentration. Consequently, vegetarian meals could decrease the rate of iron overload in IL-NTDT in comparison to meals with increased and more absorbable forms of iron from animal food products, such as red meat. ${ }^{15,26}$

Drugs may also influence the rate of iron absorption. Within this context, increased absorption of iron can be facilitated by natural or synthetic lipophilic chelators, such as maltol, 8-hydroxyquinolone, and DFX, all of which form lipophilic iron complexes. ${ }^{14,15,26,33}$ In contrast, decreased absorption of iron could be caused using hydrophilic chelators, such as DFO and $\mathrm{L}_{1}$, which form hydrophilic and/or charged iron complexes, as well as by chelators forming insoluble iron complexes (Figure 2, Table 2). 14,15,26,33

The ferrokinetic, tissue-distribution, and toxicity profiles vary among the different categories of iron-loading conditions, including TM, IL-NTDT, and hereditary hemochromatosis. In normal individuals and under normal conditions, the amount of iron absorbed from the diet is equivalent to the amount of iron excreted ( $1-4 \mathrm{mg}) .{ }^{12,15,26}$ Different levels 
of increased iron absorption are observed (2.6-8.6 mg/day) in nontransfused TI patients, which in most cases can progressively over many years lead to iron overload and subsequently to tissue and organ damage. ${ }^{12-15}$ This pattern of increased rate of iron absorption from the diet, which is estimated to be up to approximately $6 \mathrm{mg} /$ day, is similar to other IL-NTDT and also in hereditary hemochromatosis patients. ${ }^{12-15}$ In comparison, it is estimated that the daily body-iron intake in TM from red blood-cell transfusions for maintaining $\mathrm{Hb}$ levels at approximately $9-10 \mathrm{~g} / \mathrm{dL}$ is approximately $20 \mathrm{mg} /$ day and from increased gastrointestinal absorption 1-6 mg/day. ${ }^{12-15,26}$

In TM patients, organ damage due to iron overload toxicity in the absence of chelation therapy appears within a few years of initiating red blood-cell transfusions or when approximately 50 units of packed red blood cells (equivalent to $10.0-12.5 \mathrm{~g}$ of iron) have been transfused. ${ }^{14,15}$ Similar levels of iron overload appear to cause organ toxicity in TI and other related IL-NTDT conditions, but at an older age. ${ }^{14,15}$

Alternative treatments to red blood-cell transfusions are generally used in early diagnosed TI and other related IL-NTDT conditions for increasing $\mathrm{Hb}$ levels. Within this context, early diagnosed nontransfused TI patients with low $\mathrm{Hb}$ levels are currently treated with hydroxyurea, which appears to be effective in increasing $\mathrm{HbF}$ levels in some TI genotypes. ${ }^{28,34,35}$ The response to hydroxyurea is variable, and iron-loading, pharmacogenomic, and other factors appear to influence its efficacy. ${ }^{28,34-36}$ However, in many cases, and especially in older TI patients, transfusions may be initiated because of ineffective hydroxyurea treatment and increasing anemia. Erythropoietin has also been tested for elevating $\mathrm{Hb}$ levels, but the results were not satisfactory and further development in this area was not pursued. ${ }^{37}$

In undiagnosed and untreated IL-NTDT patients, excess toxic iron is accumulated over the years, leading to organ damage, including mainly liver cirrhosis, hepatocellular carcinoma, cardiomyopathy, and diabetes. During the progress of the disease, excess iron gradually accumulates initially in the liver and later to other organs, leading to an increase in serum iron, transferrin iron saturation, and serum ferritin and hepatic iron concentration, usually before the age of 30 years. In many cases, tissue damage can be diagnosed by the age of 30 years and liver cirrhosis by the age of 40 years. However, there is great variability in the progress and development of iron overload and organ damage among IL-NTDT patients and other related conditions. ${ }^{38}$ For example, Hb levels, dietary habits, hydroxyurea treatment, and many other factors can lead to different rates of iron absorption and iron overload-related tissue damage, which overall can influence the progress of the disease in IL-NTDT.

Within this context, the early diagnosis and treatment of TI and related IL-NTDT could prevent iron-overload toxicity, related tissue and organ damage, and overall decrease the morbidity and mortality in these conditions. Normal life expectancy is expected for early diagnosed and treated IL-NTDT patients who have not developed permanent organ damage, such as liver cirrhosis, cardiomyopathy, and diabetes. ${ }^{24}$

Reduction of body-iron load in IL-NTDT and other related conditions of iron overload due to increased gastrointestinal iron absorption, as well as transfusional iron overload in TM, can only be accomplished using chelation therapy and specifically the iron-chelating drugs $\mathrm{DFO}, \mathrm{L}_{1}$, and DFX (Figure 1). ${ }^{14,24}$

\section{Properties and effects of chelators and chelating drugs in relation to iron absorption}

Chelating drugs and chelators in general have different properties that can affect iron excretion as well as absorption, and can play a major role in the management of many ironmetabolism disorders, including TM, TI, and other IL-NTDT. In each case, a risk-benefit assessment is required for the selected therapies, based on individual variations and the long-term efficacy, safety, and cost of the chelating drug.

The pharmacological, toxicological, biochemical, chemical, and iron-binding properties of different chelators and the chelating drugs DFO, $\mathrm{L}_{1}$, and DFX, including their effects on iron absorption and excretion, have been previously reviewed (Table 3). ${ }^{14,26,39} \mathrm{~L}_{1}$ is a bidentate chelator forming a $3 \mathrm{~L}_{1}-1 \mathrm{Fe}$ complex, DFX is a tridentate chelator forming a $2 \mathrm{DFX}-1 \mathrm{Fe}$ complex, and DFO is a hexadentate chelator forming a $1 \mathrm{DFO}-1 \mathrm{Fe}$ complex at physiological $\mathrm{pH}$.

The mode of action and effects of chelators are variable in relation to different metabolic pathways, which is a reflection of the differences in their physicochemical and other properties (Table 3 ). This variability has been shown in many in vitro, in vivo, and clinical studies. For example, the lipid-water partition coefficient of the chelators and their iron complexes has been shown to affect the transfer of iron across the cell membrane in different cell types, as well as to cause variable effects related to the intracellular iron-metabolism pathways in these cells. ${ }^{40-42}$

In studies on the effect of chelators on iron absorption, it was observed that the long-term oral administration of lipophilic chelators, such as 8-hydroxyquinoline, appears to cause iron overload in rats, by gradually increasing the absorption 
Table 3 Mode of action and property differences of the chelating drugs deferoxamine, deferiprone, and deferasirox and other chelators*

\section{Clinical and biological effects}

Recommended doses of the chelating drugs in non-transfusiondepenent thalassemias

DFO subcutaneously or intravenously $10-60 \mathrm{mg} / \mathrm{kg} /$ day. Oral L, $10-100 \mathrm{mg} / \mathrm{kg} / \mathrm{day}$. Oral DFX $10-40 \mathrm{mg} / \mathrm{kg} /$ day. Combination of chelating drugs at different doses

Iron-loaded patient compliance with chelating drugs

Low compliance with DFO in comparison to oral $\mathrm{L}_{\text {, }}$ and DFX

Effect of chelating drugs on iron absorption

Increase of iron absorption by the lipophilic chelators maltol,

8-hydroxyquinoline, and DFX. Decrease of iron absorption by the hydrophilic chelators DFO, EDTA, DTPA, and L,

Iron removal from diferric transferrin in iron-loaded patients

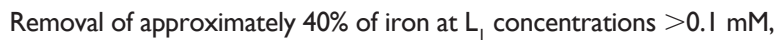

but no removal of iron by DFO or DFX at even $4 \mathrm{mM}$ concentrations

Differential iron removal from various organs of iron-loaded patients Efficacy is related to dose for all chelators. $L_{\text {, }}$ preferential iron removal from the heart and DFX from the liver. DFO iron removal from the liver and/or heart (especially following intravenous administration)

Iron redistribution in diseases of iron metabolism by chelating drugs $L_{1}$ and to a lesser extent DFO can cause iron redistribution from iron deposits and also through transferrin (only in the case of $L_{1}$ ), eg, from the reticuloendothelial system to the erythron in the anemia of chronic disease. DFX may cause redistribution of iron from the liver to other organs in iron-loaded patients. Enterohepatic circulation by DFX and DFX metabolites

Increase excretion of metals other than iron, eg, $\mathrm{Zn}$ and $\mathrm{Al}$ DTPA $>L_{1}>$ DFO (order of increased $Z n$ excretion in ironloaded patients)

DFO and $L_{1}$ cause increased $A l$ excretion in renal dialysis patients DFX causes increases in $\mathrm{Al}$ and other toxic metal absorption

Iron mobilization and excretion of chelator metabolite iron complexes Several DFO metabolites have iron-chelation potential and increase

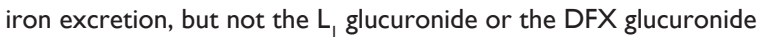
metabolites

Combination chelation therapy

$\mathrm{L}_{\text {l, }}$ DFO, and DFX combinations are more effective in iron excretion than monotherapy. The ICOC $L_{1}$ and DFO combination protocol causes normalization of the iron stores in thalassemia major and IL-NTDT patients

Chelating drug synergism with reducing agents Ascorbic acid acts synergistically with DFO but not with $L_{\text {, }}$ or DFX for increasing iron excretion

Metabolism and pharmacokinetics

Metabolite(s)

DFO: a number of metabolites, including some with chelating properties, which are cleared mainly through the urine. $L_{1}$ : the $\mathrm{L}_{1}$-glucuronide conjugate is cleared through the urine, but has no iron-chelation properties. DFX: the DFX-glucuronide conjugates are cleared through the feces and have no iron-chelation properties

$t_{1 / 2}$ absorption following oral administration

$L_{1}: 0.7-32$ minutes. DFX: $I-2$ hours

$\mathrm{T}_{\max }$ of the chelator

$\mathrm{L}_{1}$ : mostly within I hour. DFX: mostly 4-6 hours

$t_{1 / 2}$ elimination of chelator

Intravenous DFO: 5-10 minutes. Oral $L_{1}: 47-134$ minutes at $35-71 \mathrm{mg} / \mathrm{kg}$. Oral DFX: $19 \pm 6.5$ hours at 20 and $40 \mathrm{mg} / \mathrm{kg}$

(Continued)
Table 3 (Continued)

$t_{1 / 2}$ elimination of the iron complex

DFO: 90 minutes. L, : estimated within 47-134 minutes. DFX:

$17.2 \pm 7.8$ hours at $20 \mathrm{mg} / \mathrm{kg}$ and $17.7 \pm 5.1$ hours at $40 \mathrm{mg} / \mathrm{kg}$

$\mathrm{T}_{\max }$ of the iron complex

$L_{1}$ : estimated within I hour. DFX: I-6 hours at $20 \mathrm{mg} / \mathrm{kg}$ and $4-8$

hours at $40 \mathrm{mg} / \mathrm{kg}$

$\mathrm{T}_{\max }$ of the metabolite

$\mathrm{L}_{1}$-glucuronide $\mathrm{I}-3$ hours

Route of elimination of chelator and its iron complex DFO: urine and feces. $L_{1}$ : urine. DFX: almost exclusively in feces, and less than $0.1 \%-8 \%$ in urine

Enterohepatic circulation

DFX and metabolites, but not DFO or $\mathrm{L}_{1}$

Chemical and physicochemical properties

Molecular weight of chelators ( $\mathrm{g} / \mathrm{mole})$

DFO: 56I. L: 139. DFX: 373

Molecular weight of iron complexes (g/mole)

DFO: 617. L, : 470. DFX: 798

Charge of chelators at $\mathrm{pH} 7.4$

DFO: positive. $\mathrm{L}_{1}$ : neutral. DFX: negative

Charge of iron complexes at $\mathrm{pH} 7.4$

DFO: positive. $\mathrm{L}_{1}$ : neutral. DFX: negative

Partition coefficient of chelators ( $\mathrm{n}$-octanol/water)

DF: $0.02 . L_{1}: 0.19$. DFX: 6.3

$\log _{\beta}$ of chelator iron complexes DFO: 3I. L, : 35. DFX: 27

Notes: *The clinical, biological, toxicological, and pharmacological effects of ironchelating drugs and other chelators depend on their chemical and physicochemical properties, as well as the properties of their iron and other metal complexes, and also on their metabolites. There is wide variation in the mode of action of the three chelating drugs DFO, $L_{1}$, and DFX, including their efficacy in iron removal from different organs and also in the toxic side effects in iron-loaded patients, including IL-NTDT patients. Abbreviations: DFO, deferoxamine; $L_{1}$, deferiprone; DFX, deferasirox; EDTA, ethylenediaminetetraacetic acid; DTPA, diethylenetriaminepentaacetic acid; ICOC, International Committee on Chelation; IL-NTDT, iron-loaded non-transfusiondependent thalassemia.

of iron present in the diet. ${ }^{43}$ An increase in iron absorption in mice has also been shown by other lipophilic chelators, such as maltol and 2-hydroxy-4-methoxypyridine-1-oxide. ${ }^{33}$ The maltol-iron complex is currently undergoing clinical development for general use in iron deficiency and especially in patients with inflammatory bowel disease. ${ }^{44-46}$ The same effect is anticipated by the chronic administration of other lipophilic oral iron chelators and especially the chelating drug DFX. ${ }^{47,48}$ DFX is also anticipated to increase aluminum and other toxic metal absorption (Figure 2, Table 3).47,48

In contrast to maltol, DFX, and other lipophilic chelators, many hydrophilic chelators, including DFO and $\mathrm{L}_{1}$, appear to cause a decrease in iron absorption under similar conditions. ${ }^{33}$ Because of these differences in properties, $\mathrm{DFO}$ and $\mathrm{L}_{1}$ could also be used in the treatment of acute oral iron poisoning. ${ }^{49,50}$ In contrast, the use of DFX in the treatment of acute iron poisoning is inappropriate and not recommended, because it may increase the absorption of iron and increase further iron toxicity in this condition. ${ }^{47,48}$

Inhibition of iron absorption has also been observed by such chelators as 2-hydroxypyridine-1-oxide, which form 
complexes with iron that precipitate and become insoluble..$^{33}$ Increase or inhibition of iron absorption can also be observed with commonly used drugs with chelating properties, such as tetracycline and hydroxyurea. ${ }^{51,52}$ It is anticipated that similar chelators could be used to decrease the rate of gastrointestinal iron absorption in TI, other IL-NTDT, hereditary hemochromatosis, and TM.

\section{Clinical use and efficacy of deferoxamine, deferiprone, and deferasirox in iron overload}

The primary use of DFO, $\mathrm{L}_{1}$, and DFX is the treatment of transfusional iron overload in TM, other transfusional ironloaded conditions, and IL-NTDT. Iron-overload toxicity from chronic transfusions and/or increased gastrointestinal iron absorption leads to progressive multiorgan damage and associated increase in morbidity and mortality, which in most cases is directly related to the level of excess iron load. For example, in the absence of chelation therapy, regularly transfused TM patients die by the age of 20 years, mainly from congestive cardiac failure caused by cardiac iron-overload toxicity. ${ }^{53,54}$ In contrast, the mean survival of TM patients in the UK treated with DFO was estimated to be 35 years. ${ }^{54}$ However, the use of specific chelation-therapy protocols with $\mathrm{L}_{1}$ and the $\mathrm{L}_{1}-\mathrm{DFO}$ combination appear to increase life expectancy and reduce the morbidity of iron-loaded patients to levels approaching those of normal individuals..$^{24,55}$

DFO has been used parenterally for the treatment of ironoverloading conditions, including TI, other IL-NTDT, and especially TM in the last 50 years. Similarly, oral $\mathrm{L}_{1}$, which is a generic drug like DFO, has been approved and used in India since 1995 and in Europe and other regions since 1999, but in the US since only recently - in 2011. DFX is a relatively new oral but very expensive patented drug that has been used worldwide since 2005, and the patent expires in 2017.

The three chelating drugs have major differences in the efficacy, tolerance, site of action, toxicity profile, and cost, all of which affect the morbidity and mortality of iron-overloaded patients in both developed and developing countries (Table 3). ${ }^{14,24,25}$ Because of the high cost of the transfusions and chelating drugs, most of the TM and IL-NTDT patients in developing countries are left to die untreated. The local manufacturing of cheaper formulations for $\mathrm{L}_{1}$ and DFX in developing countries, such as India, Thailand, and Iran, has substantially reduced the cost of chelating drugs and increased the number of patients receiving chelation therapy in these countries. ${ }^{14,24,25,56,57}$

There are general variations among patients not only related to the level of iron overload and organ distribution but also in the response and pharmacology to each of the chelating drugs. These responses are also related to individual profile differences in the absorption, distribution, metabolism, excretion, and toxicity of the drugs. ${ }^{24,25,58,59}$ For example, $\mathrm{L}_{1}$ causes an increase in iron excretion exclusively in the urine, DFX exclusively in the feces, and DFO mostly in the urine and also the feces (Table 3). ${ }^{14,24,25,59}$

The general recommended doses of the chelating drugs in TM and IL-NTDT are 40-60 mg/kg/day for subcutaneous DF, $75-100 \mathrm{mg} / \mathrm{kg} /$ day for oral $\mathrm{L}_{1}$, and $20-40 \mathrm{mg} / \mathrm{kg} /$ day for oral DFX. However, variations in iron overload and targeted therapies can result in the application of a range of therapeutic protocols from an intensive chelation, eg, combinations of $\mathrm{DF}(40-60 \mathrm{mg} / \mathrm{kg} /$ day $)$ and $\mathrm{L}_{1}(75-100 \mathrm{mg} / \mathrm{kg} /$ day $)$ in heavily transfused iron-loaded patients, to much lower doses or intermittent withdrawal of chelation in TM and IL-NTDT patients whose normal iron-store levels have been achieved and further chelation may cause iron deficiency and other complications. ${ }^{24,60}$

In general, the selection of the chelation-therapy protocol in each iron-loaded patient depends on several other factors in addition to the iron load, such as drug efficacy, organ targeting, toxicity, cost, and compliance. However, there are many controversies in the selection and use of chelating drugs in TM and IL-NTDT that arise mainly from commercial influence, the lack of consensus in the ultimate goal or aim of the chelation therapy, and the selective use of each of the chelating drugs for optimal therapy. ${ }^{23,25}$ Furthermore, there is no consensus in the evaluation criteria and riskbenefit assessment for the use of each of the chelating drugs in personalized medicine. ${ }^{23,25}$ In most Western and other developed countries, the selection of the chelating drug for the treatment of TM and IL-NTDT depends on the choice of the physician and the commercial influence of pharmaceutical companies. ${ }^{24,25}$ In developing countries, where most patients live, the main issue is the cost of the chelating drug and its availability and not necessarily risk-benefit assessment issues. These issues have been recently highlighted within the broader context of the use of orphan drugs, which include DFO, $\mathrm{L}_{1}$, and DFX, and also of orphan and rare diseases, which include TM and IL-NTDT. ${ }^{24,25}$

The monitoring of efficacy of different chelation-therapy protocols on the gross body as well as individual organ iron-load levels has been recently achieved following the introduction of new diagnostic techniques, such as MRI $\mathrm{T}_{2}$ and $\mathrm{T}_{2}{ }^{*}$. These methods have increased our understanding of iron-metabolism and chelation pathways of iron removal and resulted in improved drug-targeting therapies of the gross body, as well as focal iron deposits in organs, which 
are the cause of toxic side effects. ${ }^{61-64}$ Furthermore, these developments have increased the prospects of the introduction of personalized medicine in TM, IL-NTDT, and other iron-metabolism disorders. ${ }^{23}$ The use of these new diagnostic methods in combination with serum ferritin monitoring made it possible to introduce chelation-therapy protocols of $\mathrm{L}_{1}$ or the $\mathrm{L}_{1}-\mathrm{DFO}$ combination that can achieve the complete elimination of iron overload in the heart and liver of almost all categories of iron-loaded patients. ${ }^{65-67}$

The clearance of excess iron in both organs can be achieved in most TM cases in 0.5-1.5 years using the International Committee on Chelation protocol on $\mathrm{L}_{1}(75-100 \mathrm{mg} / \mathrm{kg} /$ day $)$ and DFO (40-60 mg/kg/day at least 3 days per week). The clearance of excess iron from these organs is faster when using higher overall doses of $\mathrm{L}_{1}$ and DFO and in patients who are less heavily iron-loaded. ${ }^{65-69}$ The removal of excess toxic iron in TM by $\mathrm{L}_{1}$ and the $\mathrm{L}_{1}-\mathrm{DFO}$ combination is accompanied by improvement in organ function, such as elevation of left ventricular ejection fraction (LVEF) and other cardiac and endocrinological benefits. ${ }^{24,65,66,69}$ In contrast, excess iron removal from the heart by DFX is not effective and in most cases very slow, with no improvement in LVEF, especially in heavily iron-loaded TM patients. ${ }^{70}$ It should be noted that even after 5 years of treatment with DFX (30-40 mg/ $\mathrm{kg} /$ day), excess toxic amounts of iron remain in the heart of heavily iron-loaded TM patients and with no improvement in LVEF. $^{70,71}$

Excess iron removal from the liver by DFX appears to be effective usually at high doses and equivalent to that caused by $\mathrm{DFO}, \mathrm{L}_{1}$, and their combination at effective doses. ${ }^{65-72}$ The most effective and rapid clearance of excess liver iron, which is also used for intensive chelation in heavily ironloaded patients, is intravenous DFO in combination with $\mathrm{L}_{1}$. Following the clearance of excess iron from the liver and heart and the normalization of the iron stores, which is characterized by normal levels of MRI $\mathrm{T}_{2}$ and $\mathrm{T}_{2} *$ and serum ferritin, normal levels of iron are usually maintained using much lower overall chelation doses and in particular $\mathrm{L}_{1}$ monotherapy. ${ }^{65-67}$

\section{Toxicity aspects and limitations in the use of chelating drugs in iron- loaded non-transfusion-dependent thalassemias and other patient categories}

Patients with IL-NTDT and TM are regularly monitored for iron toxicity and other effects caused by their underlying condition. $^{9-11,24,73}$ Clinical examination and biochemical tests are regularly carried out for organ function, including liver-enzyme levels, echocardiography, serum ferritin, and serum Zn levels. ${ }^{9-11,16,17,24,73}$

The task of treating iron overload in IL-NTDT is generally easier in comparison to TM, since IL-NTDT patients are not as heavily iron-loaded as TM patients and also the rate of iron loading in IL-NTDT in general is much slower than that in TM. However, the prospect of chelator toxicity is a major issue, and increases especially in patients with low iron stores. ${ }^{24,60}$ Regular monitoring and prophylactic measures for chelating drug toxicity are generally recommended for these categories of patients. ${ }^{24,74}$

The treatment of patients with TM, IL-NTDT, and other categories of iron-loading conditions with normal iron stores or not heavily iron-loaded is restricted for DFX, due to toxicity implications, as underlined by the labeling information for its use by the manufacturers of the drug. ${ }^{75,76}$ In particular, the use of DFX is not recommended for ironloaded patients with serum ferritin lower than $500 \mu \mathrm{g} / \mathrm{L} .{ }^{75,76}$ This limitation is important for the safety of many patients and in particular the vast majority of IL-NTDT, who are not heavily iron-loaded and have serum ferritin lower than $500 \mu \mathrm{g} / \mathrm{L} .{ }^{75-76}$

High morbidity and mortality have been reported in different categories of patients, and especially non-iron-loaded categories of patients who have been treated with DFX. ${ }^{77,78}$ Many toxic side effects, such as fatal renal, liver, and bone marrow failure, including agranulocytosis, as well as renal toxicity, skin rashes, and gastric intolerance, have also been reported in iron-loaded patients treated with DFX..$^{74-78}$

Patients treated with DFX are regularly monitored for kidney function, and withdrawal of the drug is recommended for patients with persistent increase in serum creatinine levels. The same limitations and restrictions that apply in the use of DFX in patients with low iron stores also apply for the use of DFO, despite the incidence of serious toxic side effects being much lower in the latter. Within this context, the use of DFO in IL-NTDT and other similar categories of not heavily iron-loaded patients, as well as other categories of patients with normal iron stores, is restricted, due to toxicity implications. Fatal cases of mucormycosis, acute respiratory distress syndrome, and Yersinia enterocolitica was reported in different categories of patients treated with DFO. ${ }^{39,79}$ Furthermore, ocular and auditory toxicity has also been reported in non-heavily iron-loaded TM patients using DFO. ${ }^{39,80,81}$

In contrast, the safety of $\mathrm{L}_{1}$ in TM, IL-NTDT, and other categories of patients with low or normal iron stores has been shown during the regular use of the drug in thousands of patients in the last 20 years and also in studies in TM patients 
with normal iron stores exceeding 100 patient-years. ${ }^{24,65,67,74}$ The prospect of wider clinical use of $\mathrm{L}_{1}$ as a universal antioxidant in non-iron-overloading diseases, such as neurodegenerative, cardiovascular, renal, and infectious diseases, as well as other diseases, including cancer and aging diseases, has been investigated in clinical trials involving many of these categories of patients. ${ }^{24,65,67,74}$ In particular, the introduction of $\mathrm{L}_{1}$ for the treatment of non-iron-loaded patients by targeting focal toxic iron deposits, eg, in Friedreich's ataxia, and also of toxic labile iron, eg, in diabetic and nondiabetic glomerular disease, is a reflection of the antioxidant and safety potential of this drug. ${ }^{24,82,83}$ In these two non-iron-loaded conditions, $\mathrm{L}_{1}$ was used at doses of 50-75 mg/kg/day for 6-9 months with no serious toxic side effects. ${ }^{82,83}$ Similarly, the safety of $\mathrm{L}_{1}$ in many other categories of non-iron-loaded diseases has also been confirmed in clinical trials involving patients with normal iron stores, including the anemia of chronic disease, renal dialysis, infections, Parkinson's, and other neurodegenerative diseases. ${ }^{24,65,67,74}$

The most serious toxic side effects of $\mathrm{L}_{1}$ are reversible agranulocytosis $(<1 \%)$ and neutropenia $(<5 \%){ }^{24,84}$ Weekly or fortnightly mandatory blood count is recommended for patients using $\mathrm{L}_{1}$ for prophylaxis against this toxicity. A few fatal cases of agranulocytosis in TM have been reported for patients treated with $\mathrm{L}_{1}$ who did not follow this safety procedure. ${ }^{24,39,84}$ Less serious toxic side effects include gastric intolerance, joint pains, and Zn deficiency. $\mathrm{Zn}$ supplements are used for prophylaxis for patients on long-term treatment with $\mathrm{L}_{1}$ and DFO, including TM and IL-NTDT patients. ${ }^{24,84}$

It should be noted that the rate of fatalities associated with the use of iron-chelating drugs is different. An estimated $11.7 \%$ mortality (1,935 of 16,514 patients) was reported by the European Medicines Agency for DFX, whereas for DFO and $\mathrm{L}_{1}$, the rate of mortality is less than $0.1 \%{ }^{77,78}$ Toxicity-vigilance and prophylactic measures are important steps for ensuring the safety of TM, IL-NTDT, and other iron-loaded patients treated with chelation therapy, especially when using high doses in patients with low or normal iron stores.

\section{Effective iron-chelation therapy in thalassemia intermedia and in other iron-loaded non-transfusion- dependent thalassemias}

Iron overload and related toxicity as a result of increased gastrointestinal absorption in TI and other IL-NTDT conditions was identified more than 40 years ago. ${ }^{12,13}$
Diagnostic methods, such as serum ferritin, serum iron, increased urinary excretion in response to DFO, and postmortem examination, revealed the extent of the iron load and toxicity in these categories of patients. ${ }^{12-18,85}$

Iron-chelation therapy has been widely and successfully used from the early 1980s for the treatment of iron overload in TI, other IL-NTDT, and other similar conditions. ${ }^{86}$ Initial reports indicated the efficacy of DFO in increasing iron excretion in these categories of patients. For example, increased urinary iron excretion in response to continuous 12-hour subcutaneous DFO (20-100 mg/kg/day, maximum $2 \mathrm{~g} /$ day) and decrease in serum ferritin (initial $68-940 \mu \mathrm{g} / \mathrm{L}$ and final $48-420 \mu \mathrm{g} / \mathrm{L}$ ) after 6 months of treatment was observed in ten TI patients (age 1-17 years, five males, two splenectomized) with different $\mathrm{Hb}$ levels (7-10.5 g/dL) and variable iron load. ${ }^{86}$ Four patients participating in the study had previous transfusions to a total in each case of $0.5,1.5,2.0$, and 10.0 red blood-cell units, respectively. Dose adjustment was carried out by assessing 24-hour urinary iron excretion in response to the different doses of DFO, which were administered over 3 days. Different levels of iron load were detected in this cohort of young TI patients, including a 5 -year-old patient. A tailored chelation therapy was recommended using this DFO-response method for reducing chelator toxicity. It was also suggested that iron-chelation treatment should begin from an early age to prevent iron accumulation. ${ }^{86}$

Many nontransfused iron-loaded patients have also been treated with DF. In one case, subcutaneous DFO (2 g/day for 9-11 months) was shown to be effective in hereditary hemochromatosis patients where phlebotomy was not possible. ${ }^{87}$ Monitoring of liver iron by repeated noninvasive liver ironconcentration measurements using superconducting quantum interference-device biosusceptometry has shown that DFO was as effective in liver-iron elimination (12 mg/day) as normal phlebotomy treatment $(14.3 \mathrm{mg} /$ day $)$ of weekly $500 \mathrm{~mL}$ blood removals. ${ }^{87}$

Successful iron-chelation treatments in TI and other ILNTDT patients have also been reported using $\mathrm{L}_{1}$. For example, $\mathrm{L}_{1}$ at low doses of $25-50 \mathrm{mg} / \mathrm{kg} /$ day was studied for 17 weeks and 86 weeks in seven $\mathrm{HbE} \beta$-thalassemia and two TM ironloaded nontransfused patients. ${ }^{88}$ Substantial reductions in serum ferritin (from mean 2,168 to $418 \mu \mathrm{g} / \mathrm{L}$ ) and hepatic iron (from mean 20.3 to $11.7 \mathrm{mg} / \mathrm{g}$ dry weight) were observed in these patients. ${ }^{88}$ Similar results were obtained in another study in 16 NTDT HbH disease (3/4 $\alpha$-globin gene deletion) patients (29-76 years) using $\mathrm{L}_{1}$ at $50 \mathrm{mg} / \mathrm{kg} /$ day initially for 4-8 months. The dose then increased in the majority of cases to $75 \mathrm{mg} / \mathrm{kg} /$ day. ${ }^{89}$ In 18 months, mean serum ferritin 
decreased from 1,492 to $519 \mu \mathrm{g} / \mathrm{L}$, and in nine of the patients was below the normal cutoff point of $397 \mu \mathrm{g} / \mathrm{L}$ before the 18-month monitoring period. Significant improvement in cardiac function and decrease in liver-iron content measured by MRI $\mathrm{T}_{2}$ was also detected in 12 of the patients. ${ }^{89}$ No reduction in iron load was observed in a control group of 16 patients with $\mathrm{HbH}$ disease who received no chelation treatment in the same study. ${ }^{89}$

The use of higher doses of $\mathrm{L}_{1}(75-100 \mathrm{mg} / \mathrm{kg} /$ day) in ILNTDT can result in the rapid normalization of iron stores. For example, administration of $\mathrm{L}_{1}$ at $75 \mathrm{mg} / \mathrm{kg}$ /day for 9 months in an iron-loaded, nontransfused TI patient with cardiac and other problems caused a substantial reduction to normal levels in serum ferritin (from 2,174 to $251 \mu \mathrm{g} / \mathrm{L}$ ) and hepatic iron (from 14.6 to $1.9 \mathrm{mg} / \mathrm{g}$ dry weight). ${ }^{90}$ An increase in cardiac and liver MRI $\mathrm{T}_{2}$ relaxation time was also observed following the 9-month treatment, indicating the effective removal of iron from these organs by $\mathrm{L}_{1} \cdot{ }^{90}$

In a 5-year long-term randomized clinical trial of 88 occasionally transfused TI patients (median age 41 years, 48 females $)$, the effectiveness of oral $\mathrm{L}_{1}(50-75 \mathrm{mg} / \mathrm{kg} /$ day $)$ on 47 patients with median serum ferritin $1,221 \mu \mathrm{g} / \mathrm{L}$ was compared to a group of 41 patients with median serum ferritin $1,122 \mu \mathrm{g} / \mathrm{L}$ treated with subcutaneous DFO (30-50 mg/kg/day, 5 days per week). ${ }^{91}$ Both chelators were found to be effective in reducing iron load, with median serum ferritin levels reaching $579 \mu \mathrm{g} / \mathrm{L}$ in the $\mathrm{L}_{1}$ group and $484 \mu \mathrm{g} / \mathrm{L}$ in the DFO group. It was concluded in this $>200$ patient-year-long study that $\mathrm{L}_{1}$ was associated with an efficacy and safety profile similar to that of DFO. ${ }^{91}$ Toxic side effects of $\mathrm{L}_{1}$ were gastric intolerance, joint pains, neutropenia in six, and agranulocytosis in four patients. Compliance was $85 \%$ for patients on $\mathrm{L}_{1}$ and $76 \%$ for DFO. The cost of chelation between $\mathrm{L}_{1}$ and DF was not significantly different, and was estimated to be about ten times lower than that of DFX. ${ }^{56,57,91}$ A follow-up 5-year trial using $\mathrm{L}_{1}$ and DFO continued with members of the same group of TI patients, with similar results. ${ }^{91}$

Rapid clearance of excess iron in iron-loaded nontransfused patients has also been observed using the $\mathrm{L}_{1}-\mathrm{DFO}$ combination, which appears to be easily achieved and also to be faster in comparison to TM patients. ${ }^{65-69}$ For example, a combination of intravenous DFO (30 mg/kg/day for 5 months) and oral $\mathrm{L}_{1}$ (75 mg/kg/day for 2 months) was used in a juvenile hemochromatosis nontransfused iron-loaded patient (serum ferritin 3,773 $\mu \mathrm{g} / \mathrm{L}$ ) with severe cardiomegaly and congestive heart failure with an ejection fraction of $25 \%{ }^{92}$ The combination $\mathrm{L}_{1}-\mathrm{DFO}$ chelation treatment caused an improvement in the ejection fraction to $49 \%$ and blood parameters, and also removal of antiarrhythmic drugs and normalization of serum ferritin. ${ }^{92}$ Increase in the ejection fraction has also been observed in different groups of TM patients using $\mathrm{L}_{1}$ and the $\mathrm{L}_{1}-\mathrm{DFO}$ combination. ${ }^{24,65-69}$ Overall, effective and safe chelation therapy using DFO, $\mathrm{L}_{1}$, and their combination could be applied to TI and other IL-NTDT patients.

A number of clinical trials have been carried out to test the effect of DFX in TI and other IL-NTDT patients. ${ }^{22}$ The efficacy and toxicity of DFX appear to be dose-dependent. For example, in a randomized 1-year clinical trial involving 110 TI patients, some NTDT and others receiving occasional transfusions, DFX (5-10 mg/kg/day) caused no significant change in serum ferritin at 24 weeks and only significant change at 52 weeks at $10 \mathrm{mg} / \mathrm{kg} /$ day compared to baseline. ${ }^{93}$ Similar changes were reported for liver-iron concentration. Adverse events were reported in $78 \%$ of the patients, and only $24 \%$ were thought to be drug-related, namely nausea, rash, and diarrhea. ${ }^{93}$ In a continuation study for a further year, the results of the use of DFX (5-20 mg/kg/day) on efficacy and toxicity in a selected group of six not previously chelated of the placebo group and 18 of the 110 chelated patients (12 TI, six $\alpha$-thalassemia, and six $\mathrm{HbE} \beta$-thalassemia) were reported..$^{93}$ Median serum ferritin before the study was 825 (range: $393-2,169) \mu \mathrm{g} / \mathrm{L}$, and by the end of the study had decreased to 427 (range: 102-1,010) $\mu \mathrm{g} / \mathrm{L}$. Similar changes were reported for liver-iron concentration. ${ }^{93}$ Approximately $29 \%$ of the adverse events reported were similar to the previous study and thought to be drug-related. ${ }^{93}$ In a different 1-year study involving a cohort of 50 TI patients, DFX (10-40 mg/kg/day) caused a decrease of serum ferritin from baseline mean 1,700 to $835 \mu \mathrm{g} / \mathrm{L}$ in 8 months, but thereafter remained stable or slightly increased to $880 \mu \mathrm{g} / \mathrm{L}$ on the 12th month. ${ }^{94}$

In contrast to these studies, lower efficacy and higher toxicity was reported for DFX in TI and other IL-NTDT patients by investigators not related or funded by the manufacturers of DFX. ${ }^{95}$ For example, in a retrospective study of $72 \mathrm{TI}$ and TM patients of mean age 20 years, the use of DFX (20-40 mg/kg/day) for a mean 16.7 months resulted in a $25 \%$ dropout due to toxicity and low efficacy..$^{95}$ In particular, adverse effects of renal function were reported, including a $>33 \%$ increase in serum creatinine in 43 patients. Persistent increase in serum creatinine in seven of the 43 patients caused discontinuation of DFX. ${ }^{95}$ No significant change in serum ferritin was observed at the end of the study (initial 3,059 \pm 309 versus final 2,965 $\pm 309 \mu \mathrm{g} / \mathrm{L}$ ). Similarly, no change in cardiac iron load but a decrease in liver iron was observed in this 
cohort of patients. The patients affected by the DFX toxicity reverted back to $\mathrm{L}_{1}$, DFO, or the $\mathrm{L}_{1}-\mathrm{DFO}$ combination. ${ }^{95}$ High incidence of renal toxicity using DFX has also been reported in many other groups of patients, including young patients, by independent investigators not sponsored or without connections with the manufacturers of DFX. ${ }^{96-98}$

The variation in the iron overload in patients of IL-NTDT and the restriction imposed on the safety for the use of the chelating drugs in different iron-overload levels, as well as the target iron-loaded organ(s), requires the design of specific chelation protocols for each patient case. Similar chelation protocols apply in the treatment of TM patients with equivalent iron overload to IL-NTDT patients. It appears that the selection of the most effective and safest chelation protocol together with the monitoring of the iron load using serum ferritin and $\mathrm{MRI} \mathrm{T}_{2}$ or $\mathrm{T}_{2}{ }^{*}$, as well as the use of prophylactic measures for chelating drug toxicity, could ensure the effective reduction and maintenance of iron load to normal levels and lead to an overall decrease in morbidity and mortality in IL-NTDT patients.

\section{Future therapeutic approaches of iron-chelation therapy in iron- loaded nontransfused thalassemia patients caused by increased iron absorption}

Patients with TI and other IL-NTDT have a diverse genotypic and clinical picture of variable severity, including different levels of iron overload and associated toxicity in the liver, heart, and other organs..$^{10,11,17,19,27,32,73}$ Within this context, several approaches in relation to chelation therapy could be envisaged. The design and application of optimal chelation therapies will be required in each case based on a risk-benefit assessment of the $\operatorname{drug}(\mathrm{s})$ and other factors similar to TM patients. ${ }^{23,24}$ In particular, the cost of chelating drugs is very important, since most TI and other IL-NTDT patients live in developing countries and cannot afford expensive drug treatments. ${ }^{56,57,99,100}$

DFO and $\mathrm{L}_{1}$ appear to be effective in the removal of excess iron load in TI and other IL-NTDT patients, even at doses lower than those used in TM and other transfused patients. Selected combinations of DFO and $\mathrm{L}_{1}$, such as the International Committee on Chelation protocol, could be used as a first-line treatment not only for the rapid removal of excess cardiac iron in cases of iron-overload cardiomyopathy in TI and other IL-NTDT patients but also in the rapid clearance of liver-iron overload. . $^{23,24,65,66,86}$
A critical question in relation to the treatment of iron overload in TI and other IL-NTDT is the timing of the initiation of the chelation therapy. Recent studies in TM patients suggest that the removal of iron by chelating drugs in TI and other IL-NTDT should be initiated as soon as the diagnosis is confirmed and with a major aim the complete removal of all excess iron and prevention of its accumulation in organs. ${ }^{23,24,86}$ In studies of TM patients with normalrange iron-store levels, lower overall doses of chelating drugs and in particular $\mathrm{L}_{1}$ were used for achieving this aim. ${ }^{65,67}$ Furthermore, the advantage of this approach is that in patients with normal-range iron stores, the damage to organs is prevented and clinical management can continue without related organ complications, such as liver cirrhosis and cardiomyopathy. ${ }^{23,24}$

$\mathrm{L}_{1}$ should be considered as a first-line treatment for $\mathrm{TI}$ and other IL-NTDT patients with low or normal iron stores, since it would appear from studies in these categories that included cohorts of TM patients to be safer and more effective in comparison to DFO and DFX. ${ }^{74-77}$ The latter two chelating drugs are recommended by the manufacturers to be used in patients with serum ferritin levels higher than $500 \mu \mathrm{g} / \mathrm{L} .{ }^{74-77}$ Similarly, the prophylactic use of low doses of $\mathrm{L}_{1}$ (eg, $10 \mathrm{mg} / \mathrm{kg} /$ day) may be sufficient in preventing iron overload in young and older patients with TI and other IL-NTDT.

Many safety, efficacy, and financial questions are raised regarding the use of DFX in TI and IL-NTDT patients. Additional toxicity issues can be envisaged in patients with low iron stores, where DFX may cause an increase in the absorption of dietary iron and other toxic metals, eg, aluminum. ${ }^{23,48}$ DFX is also very expensive and cannot be afforded by the vast majority of patients, especially those living in developing countries. ${ }^{3,23,56,57,99,100}$ The introduction of combinations of DFX with DFO and $\mathrm{L}_{1}$ increases the prospect of more effective and less toxic therapies for TM, TI, IL-NTDT, and other related conditions in comparison to DFX monotherapies. ${ }^{100-105}$ Pharmacological and toxicological data are important parameters for the construction of such combination therapies. ${ }^{101,106,107}$

Although current interest in TI and other IL-NTDT patients is mainly related to the marketing efforts of the manufacturers of DFX and their academic partners, there are many other fields of investigation that need to be explored in addition to iron-chelation therapy. Some of the strategies for the treatment of the other clinical complications may also influence the rate of iron overload and chelation therapy. Within this context, the time of initiation and the rate of red blood-cell transfusions, as well as splenectomy, will have 
a direct effect on iron loading and change in the chelation strategies. ${ }^{85,105}$ Similarly, the role of iron overload and chelation therapy on the activation of $\mathrm{HbF}$ production by hydroxyurea, which also has iron-chelation properties, has

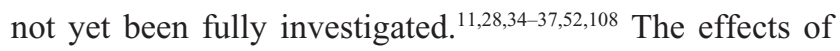
modulators of erythropoietin, hepcidin, ferroportin, and other regulatory molecules on iron metabolism and erythropoietic activity are also expected to influence future chelationtherapy strategies in TI and other IL-NTDT. ${ }^{38,109,110}$

A major therapeutic strategy in TI, other IL-NTDT, and especially hereditary hemochromatosis is the prevention or decrease of gastrointestinal iron absorption. This can be accomplished by new, synthetic, or naturally occurring iron chelators that can bind iron in the gastrointestinal tract and inhibit or decrease the rate of its transfer and storage in the body. In particular, chelators and chelatoriron complexes that are not orally absorbed and chelators that form insoluble iron complexes could be considered for such treatments. ${ }^{33,111}$ Inhibitors of DMT1 activity and activators of hepcidin production could also be used for minimizing iron absorption. The combination of chelators and of chelators with regulators of DMT1, hepcidin, and ferroportin could also be considered for potential use in reducing increased iron absorption and associated iron overload in IL-NTDT. ${ }^{33,109-111}$

The inhibition of iron absorption in IL-NTDT could be accompanied by relocation and reutilization of stored iron for erythropoiesis and other iron-metabolism activities. Within this context, $\mathrm{L}_{1}$ has been shown to facilitate this process by the redistribution of iron stored in hepatocytes and macrophages to hemopoietic tissues via iron exchange with transferrin. ${ }^{12-119}$

Overall, several treatments could become available for the future management of TI, other IL-NTDT, and hereditary hemochromatosis. These could involve the use of chelation therapies for increasing iron excretion, chelation therapies for inhibiting iron absorption, chelation therapies for iron redistribution, selected diets with lower and less absorbable forms of iron, the use of regulators of DMT1, hepcidin, and ferroportin and other proteins of iron metabolism involved in the transport of iron, activators of $\mathrm{HbF}$ production, and combinations of such treatments.

\section{Acknowledgments}

This study was supported from internal funds of the Postgraduate Research Institute of Science, Technology, Environment and Medicine, a nonprofit, charitable organization. The article was a result of an invitation by the journal editors.

\section{Disclosure}

The authors report no conflicts of interest in this work.

\section{References}

1. Pantopoulos K, Porwal SK, Tartakoff A, Devireddy L. Mechanisms of mammalian iron homeostasis. Biochemistry. 2012;51(29): $5705-5724$

2. Andrews NC. Forging a field: the golden age of iron biology. Blood. 2008;112(2):219-230.

3. [No authors listed]. Community control of hereditary anaemias: memorandum from a WHO meeting. Bull World Health Organ. 1983; 61(1):63-80.

4. Weatherall DJ, Glegg JB. The Thalassaemia Syndromes. 3rd ed. Oxford: Blackwell Scientific Publications; 1981.

5. Feder JN, Gnirke A, Thomas W, et al. A novel MHC class I-like gene is mutated in patients with hereditary haemochromatosis. Nat Genet. 1996;13(4):339-408.

6. Barton JC, Edwards CQ. Hemochromatosis: Genetics, Pathophysiology, Diagnosis and Treatment. Cambridge: Cambridge University Press; 2000.

7. Halliwell B, Gutteridge JM. Free radicals and antioxidant protection: mechanisms and significance in toxicology and disease. Hum Toxicol. 1988;7(1):7-13.

8. Kontoghiorghes GJ. Prospects for introducing deferiprone as potent pharmaceutical antioxidant. Front Biosci (Elite Ed). 2009;1: 161-178.

9. Singer ST. Variable clinical phenotypes of $\alpha$-thalassemia syndromes. ScientificWorldJournal. 2008;9:615-625.

10. Galanello R, Cao A. Relationship between genotype and phenotype: thalassemia intermedia. Ann NY Acad Sci. 1998;850:325-333.

11. Borgna-Pignatti C. Modern treatment of thalassaemia intermedia. Br J Haematol. 2007;138(3):291-304.

12. Pippard MJ, Callender ST, Warner GT, Weatherall DJ. Iron absorption and loading in $\beta$-thalassaemia intermedia. Lancet. 1979;2(8147): 819-821.

13. Pootrakul P, Kitcharoen K, Yansukon P, et al. The effect of erythroid hyperplasia on iron balance. Blood. 1988;71(4):1124-1129.

14. Kontoghiorghes GJ, Eracleous E, Economides C, Kolnagou A. Advances in iron overload therapies. Prospects for effective use of deferiprone (L1), deferoxamine, the new experimental chelators ICL670, GT56-252, L1NAll and their combinations. Curr Med Chem. 2005;12(23):2663-2681.

15. Kontoghiorghes GJ, Spyrou A, Kolnagou A. Iron chelation therapy in hereditary hemochromatosis and thalassemia intermedia: regulatory and non regulatory mechanisms of increased iron absorption. Hemoglobin. 2010;34(3):251-264.

16. Aessopos A, Kati M, Farmakis D. Heart disease in thalassemia intermedia: a review of the underlying pathophysiology. Haematologica. 2007;92(5):658-665.

17. Sonakul D, Pacharee P, Wasi P, Fucharoen S. Cardiac pathology in 47 patients with $\beta$-thalassaemia/haemoglobin E. Southeast Asian J Trop Med Public Health. 1984;15(4):554-563.

18. Aessopos A, Farmakis D, Trompoukis C, et al. Cardiac involvement in sickle $\beta$-thalassemia. Ann Hematol. 2009;88(6):557-564.

19. Aessopos A, Farmakis D, Karagiorga M, et al. Cardiac involvement in thalassemia intermedia: a multicenter study. Blood. 2001;97(11): 3411-3416.

20. Borgna-Pignatti C, Garani MC, Forni GL, et al. Hepatocellular carcinoma in thalassaemia: an update of the Italian registry. Br J Haematol. 2014;167(1):121-126.

21. Fragatou S, Tsourveloudis I, Manesis G. Incidence of hepatocellular carcinoma in a thalassemia unit. Hemoglobin. 2010;34(3): 221-226.

22. Taher AT, Porter JB, Viprakasit V, et al. Approaching low liver iron burden in chelated patients with non-transfusion-dependent thalassemia: the safety profile of deferasirox. Eur J Haematol. 2014;92(6): 521-526. 
23. Kontoghiorghes GJ. A new era in iron chelation therapy: the design of optimal, individually adjusted iron chelation therapies for the complete removal of iron overload in thalassemia and other chronically transfused patients. Hemoglobin. 2009;33(5):332-338.

24. Kolnagou A, Kontoghiorghe CN, Kontoghiorghes GJ. Transition of thalassaemia and Friedreich ataxia from fatal to chronic diseases. World J Methodol. 2014;4(4):197-218.

25. Kontoghiorghe CN, Andreou N, Constantinou K, Kontoghiorghes GJ. World health dilemmas: orphan and rare diseases, orphan drugs and orphan patients. World J Methodol. 2014;4(3):163-188.

26. Kontoghiorghes GJ, Kolnagou A. Molecular factors and mechanisms affecting iron and other metal excretion or absorption in health and disease: the role of natural and synthetic chelators. Curr Med Chem. 2005;12(23):2695-2709.

27. Fucharoen S, Ketvichit P, Pootrakul P, Siritanaratkul N, Piankijagum A, Wasi P. Clinical manifestation of $\beta$-thalassemia/hemoglobin E disease. J Pediatr Hematol Oncol. 2000;22(6):552-557.

28. Vichinsky E. Hemoglobin E syndromes. Hematology Am Soc Hematol Educ Program. 2007:79-83.

29. Pantopoulos K. Function of the hemochromatosis protein HFE: lessons from animal models. World J Gastroenterol. 2008;14(45): 6893-6901.

30. Byrnes V, Barrett S, Ryan E, et al. Increased duodenal DMT-1 expression and unchanged HFE mRNA levels in HFE-associated hereditary hemochromatosis and iron deficiency. Blood Cells Mol Dis. 2002 29(3):251-260.

31. Mavrogeni S, Gotsis E, Ladis V, et al. Magnetic resonance evaluation of liver and myocardial iron deposition in thalassemia intermedia and $\beta$-thalassemia major. Int J Cardiovasc Imaging. 2008;24(8):849-854

32. Au WY, Lam WW, Chu WW, et al. Organ-specific hemosiderosis and functional correlation in Chinese patients with thalassemia intermedia and hemoglobin H disease. Ann Hematol. 2009;88(10):947-950.

33. Kontoghiorghes GJ. Chelators affecting iron absorption in mice. Arzneimittelforschung. 1990;40(12):1332-1335.

34. Italia KY, Jijina FJ, Merchant R, et al. Response to hydroxyurea in $\beta$-thalassemia major and intermedia: experience in western India. Clin Chim Acta. 2009;407(1-2):10-15.

35. Singer ST, Kuypers FA, Olivieri NF, et al. Fetal haemoglobin augmentation in $\mathrm{E} / \boldsymbol{\beta}^{0}$ thalassaemia: clinical and haematological outcome. Br J Haematol. 2005;131(3):378-388.

36. Patrinos GP, Grosvelt FG. Pharmacogenomics and therapeutics of haemoglobinopathies. Hemoglobin. 2008;32(1-2):229-236.

37. Olivieri NF, Freedman MH, Perrine SP, et al. Trial of recombinant human erythropoietin: three patients with thalassemia intermedia. Blood. 1992;80(12):3258-3260.

38. Adekile AD, Azab AF, Al-Sharida SI, et al. Clinical and molecular characteristics of non-transfusion-dependent thalassemia in Kuwait. Hemoglobin. 2015;39(5):320-326.

39. Kontoghiorghes GJ, Neocleous K, Kolnagou A. Benefits and risks of deferiprone in iron overload in thalassaemia and other conditions: comparison of epidemiological and therapeutic aspects with deferoxamine. Drug Saf. 2003;26(8):553-584.

40. Kontoghiorghes GJ. Structure/red blood cell permeability activity of iron(III) chelator complexes. Inorganica Chim Acta. 1988;151(2):101-106.

41. Kontoghiorghes GJ, May A. Uptake and intracellular distribution of iron from transferrin and chelators in erythroid cells. Biol Met. 1990; 3(3-4):183-187.

42. Forsbeck K, Nilsson K, Kontoghiorghes GJ. Variation in iron accumulation, transferrin membrane binding and DNA synthesis in the K-562 and U-937 cell lines induced by chelators and their iron complexes. Eur J Haematol. 1987;39(4):318-325.

43. Yamamoto RS, Williams GM, Frangel HH, Weisburger JH. 8-Hydroxyquinoline:chronic toxicity and inhibitory effect on the carcinogenicity of N-2-fluorenylacetamide. Toxicol Appl Pharmacol. 1971; 19(4):687-698.

44. Kontoghiorghes GJ. The Design of Orally Active Iron Chelators for the Treatment of Thalassaemia [doctoral thesis]. Colchester, UK: University of Essex; 1982.
45. Harvey RS, Reffitt DM, Doig LA, et al. Ferric trimaltol corrects iron deficiency anaemia in patients intolerant of iron. Aliment Pharmacol Ther. 1998;12(9):845-848.

46. Gasche C, Ahmad T, Tulassay Z, et al. Ferric maltol is effective in correcting iron deficiency anemia in patients with inflammatory bowel disease: results from a phase-3 clinical trial program. Inflamm Bowel Dis. 2015;21(3):579-588.

47. Kontoghiorghes GJ. Do we need more iron-chelating drugs? Lancet. 2003;362(9382):495-496.

48. Kontoghiorghes GJ. Transparency and access to full information for the fatal or serious toxicity risks, low efficacy and high price of deferasirox, could increase the prospect of improved iron chelation therapy worldwide. Hemoglobin. 2008;32(6):608-615.

49. Dresow B, Fischer R, Nielsen P, Gabbe EE, Piga A. Effect of oral iron chelator L1 on iron absorption in man. Ann N Y Acad Sci. 1998; 850:466-468.

50. Berkovitch M, Livne A, Lushkov G, et al. The efficacy of oral deferiprone in acute iron poisoning. Am J Emerg Med. 2000;18(1):36-40.

51. Djaldetti M, Fishman P, Notti I, Bessler H. The effect of tetracycline administration on iron absorption in mice. Biomedicine. 1981; 35(5):150-152.

52. Konstantinou E, Pashalidis I, Kolnagou A, Kontoghiorghes GJ. Interactions of hydroxycarbamide (hydroxyurea) with iron and copper: implications on toxicity and therapeutic strategies. Hemoglobin. 2011; 35(3):237-246.

53. Zurlo MG, De Stefano P, Borgna-Pignatti C, et al. Survival and causes of death in thalassaemia major. Lancet. 1989;2(8653):27-30.

54. Modell B, Khan M, Darlison M. Survival in $\beta$-thalassaemia major in the UK: data from the UK Thalassaemia Register. Lancet. 2000; 355(9220):2051-2052.

55. Telfer PT, Warburton F, Christou S, et al. Improved survival in thalassemia major patients on switching from desferrioxamine to combined chelation therapy with desferrioxamine and deferiprone. Haematologica. 2009;94(12):1777-1778.

56. Kontoghiorghes GJ. Ethical issues and risk/benefit assessment of iron chelation therapy: advances with deferiprone/deferoxamine combinations and concerns about the safety, efficacy and costs of deferasirox. Hemoglobin. 2008;32(1-2):1-15.

57. Cohen AR. Iron chelation therapy: you gotta have heart. Blood. 2010; 115(12):2333-2334

58. Dézsi L, Vécsei L. Clinical implications of irregular ADMET properties with levodopa and other antiparkinson's drugs. Expert Opin Drug Metab Toxicol. 2014;10(3):409-424.

59. Kontoghiorghes GJ, Bartlett AN, Sheppard L, Barr J, Nortey P. Oral iron chelation therapy with deferiprone. Monitoring of biochemical, drug and iron excretion changes. Arzneimittelforschung. 1995;45(1): 65-69.

60. Aessopos A, Kati M, Farmakis D, Polonifi E, Deftereos S, Tsironi M. Intensive chelation therapy in $\beta$-thalassemia and possible adverse cardiac effects of desferrioxamine. Int $J$ Hematol. 2007;86(3): 212-215.

61. Mavrogeni SI, Gotsis ED, Markussis V, et al. T2 relaxation time study of iron overload in $\beta$-thalassemia. MAGMA. 1998;6(1):7-12.

62. Anderson LJ, Holden S, Davis B, et al. Cardiovascular T2-star (T2*) magnetic resonance for the early diagnosis of myocardial iron overload. Eur Heart J. 2001;22(23):2171-2179.

63. Kolnagou A, Yazman D, Economides C, Eracleous E, Kontoghiorghes GJ. Uses and limitations of serum ferritin, magnetic resonance imaging $\mathrm{T} 2$ and $\mathrm{T} 2 *$ in the diagnosis of iron overload and in the ferrikinetics of normalization of the iron stores in thalassemia using the International Committee on Chelation deferiprone/deferoxamine combination protocol. Hemoglobin. 2009;33(5):312-322.

64. Pennell DJ. T2* magnetic resonance and myocardial iron in thalassemia. Ann N Y Acad Sci. 2005;1054:373-378.

65. Kolnagou A, Kleanthous M, Kontoghiorghes GJ. Reduction of body iron stores to normal range levels in thalassaemia by using a deferiprone/ deferoxamine combination and their maintenance thereafter by deferiprone monotherapy. Eur J Haematol. 2010;85(5):430-438. 
66. Farmaki K, Tzoumari I, Pappa C, Chouliaras G, Berdoukas V. Normalisation of total body iron load with very intensive combined chelation reverses cardiac and endocrine complications of thalassaemia major. Br J Haematol. 2010;148(3):466-475.

67. Kolnagou A, Kontoghiorghes GJ. Maintenance of normal range body iron store levels for up to 4.5 years in thalassemia major patients using deferiprone monotherapy. Hemoglobin. 2010;34(3):204-209.

68. Kolnagou A, Kleanthous M, Kontoghiorghes GJ. Efficacy, compliance and toxicity factors are affecting the rate of normalization of body iron stores in thalassemia patients using the deferiprone and deferoxamine combination therapy. Hemoglobin. 2011;35(3):186-198.

69. Tanner MA, Galanello R, Dessi C, et al. Combined chelation therapy in thalassemia major for the treatment of severe myocardial siderosis with left ventricular dysfunction. J Cardiovasc Magn Reson. 2008;10:12.

70. Wood JC, Kang BP, Thompson A, et al. The effect of deferasirox on cardiac iron in thalassemia major: impact of total body iron stores. Blood. 2010;116(4):537-543.

71. Vlachaki E, Agapidou A, Spanos G, et al. Five years of deferasirox therapy for cardiac iron in $\beta$-thalassemia major. Hemoglobin. 2015; 39(5):299-304.

72. Meerpohl JJ, Antes G, Rücker G, et al. Deferasirox for managing iron overload in people with thalassaemia. Cochrane Database Syst Rev. 2012;2:CD007476.

73. Wasi P, Pootrakul P, Fucharoen S, Winichagoon P, Wilairat P, Promboon A. Thalassemia in southeast Asia: determination of different degrees of severity of anemia in thalassemia. Ann N Y Acad Sci. 1985; 445:119-126

74. Kontoghiorghes GJ, Kolnagou A, Peng CT, Shah SV, Aessopos A. Safety issues of iron chelation therapy in patients with normal range iron stores including thalassaemia, neurodegenerative, renal and infectious diseases. Expert Opin Drug Saf. 2010;9(2):201-206.

75. US Food and Drug Administration. Clinical review: Exjade (deferasirox, ICL-670). 2005. Available from: http://www.fda.gov/ohrms/dockets/ ac/05/briefing/2005-4177B1_02_b.pdf. Accessed December 1, 2015.

76. Exjade (deferasirox) tablets for oral suspension [prescribing information]. East Hanover, NJ: Novartis Pharmaceutical Corporation; 2011. Available from: http://www.accessdata.fda.gov/drugsatfda_docs/ label/2010/021882s010lbl.pdf. Accessed December 1, 2015.

77. Kontoghiorghes GJ. A record number of fatalities in many categories of patients treated with deferasirox: loopholes in regulatory and marketing procedures undermine patient safety and misguide public funds? Expert Opin Drug Saf. 2013;12(5):605-609.

78. Kontoghiorghes GJ. Turning a blind eye to deferasirox's toxicity? Lancet. 2013;381(9873):1183-1184.

79. Boelaert JR, Fenves AZ, Coburn JW. Deferoxamine therapy and mucormycosis in dialysis patients: report of an international registry. Am J Kidney Dis. 1991;18(6):660-667.

80. Orton RB, de Veber LL, Sulh HM. Ocular and auditory toxicity of longterm, high-dose subcutaneous deferoxamine therapy. Can JOphthalmol. 1985;20(4):153-156.

81. Cases A, Kelly J, Sabater F, et al. Ocular and auditory toxicity in hemodialyzed patients receiving desferrioxamine. Nephron. 1990; 56(1):19-23.

82. Boddaert N, Sang KH, Rötig A, et al. Selective iron chelation in Friedreich ataxia: biologic and clinical implications. Blood. 2007; 110(1):401-408

83. Rajapurkar MM, Hegde U, Bhattacharya A, Alam MG, Shah SV. Effect of deferiprone, an oral iron chelator, in diabetic and non-diabetic glomerular disease. Toxicol Mech Methods. 2013;23(1):5-10.

84. Cohen AR, Galanello R, Piga A, De Sanctis V, Tricta F. Safety and effectiveness of long-term therapy with the oral iron chelator deferiprone. Blood. 2003;102(5):1583-1587.

85. Pootrakul P, Vongsmasa V, Laongpanich P, Wasi P. Serum ferritin levels in thalassemias and the effect of splenectomy. Acta Haematol. 1981; 66(4):244-250.

86. Cossu P, Toccafondi C, Vardeu F, et al. Iron overload and desferrioxamine chelation therapy in $\beta$-thalassemia intermedia. Eur J Pediatr. 1981;137(3):267-271.
87. Nielsen P, Fischer R, Buggisch P, Janka-Schaub G. Effective treatment of hereditary haemochromatosis with desferrioxamine in selected cases. Br J Haematol. 2003;123(5):952-953.

88. Pootrakul P, Sirankapracha P, Sankote J, et al. Clinical trial of deferiprone iron chelation therapy in $\beta$-thalassaemia/haemoglobin $E$ patients in Thailand. Br J Haematol. 2003;122(2):305-310.

89. Chan JC, Chim CS, Ooi CG, et al. Use of the oral chelator deferiprone in the treatment of iron overload in patients with $\mathrm{Hb} \mathrm{H}$ disease. Br J Haematol. 2006;133(2):198-205.

90. Olivieri NF, Koren G, Matsui D, et al. Reduction of tissue iron stores and normalization of serum ferritin during treatment with the oral iron chelator L1 in thalassemia intermedia. Blood. 1992; 79(10):2741-2748.

91. Calvaruso G, Vitrano A, Di Maggio R, et al. Deferiprone versus deferoxamine in thalassemia intermedia: results from a 5-year long-term Italian multicenter randomized clinical trial. Am J Hematol. 2015; 90(7):634-638.

92. Fabio G, Minonzio F, Delbini P, Bianchi A, Cappellini MD. Reversal of cardiac complications by deferiprone and deferoxamine combination therapy in a patient affected by a severe type of juvenile hemochromatosis (JH). Blood. 2007;109(1):362-364.

93. Taher AT, Porter J, Viprakasit V, et al. Deferasirox reduces iron overload significantly in nontransfusion-dependent thalassemia: 1 -year results from a prospective, randomized, double-blind, placebocontrolled study. Blood. 2012;120(5):970-977.

94. Karimi M, Arandi N, Haghpanah S, et al. Efficacy of deferasirox $\left(\right.$ Exjade $^{\mathbb{R}}$ ) in modulation of iron overload in patients with $\beta$-thalassemia intermedia. Hemoglobin. 2015;39(5):327-329.

95. Al-Khabori M, Bhandari S, Al-Huneini M, Al-Farsi K, Panjwani V, Daar S. Side effects of deferasirox iron chelation in patients with beta thalassemia major or intermedia. Oman Med J. 2013;28(2): $121-124$.

96. Dee CM, Cheuk DK, Ha SY, Chiang AK, Chan GC. Incidence of deferasirox-associated renal tubular dysfunction in children and young adults with $\beta$-thalassaemia. Br J Haematol. 2014;167(3):434-436.

97. Chuang GT, Tsai IJ, Tsau YK, Lu MY. Transfusion-dependent thalassaemic patients with renal Fanconi syndrome due to deferasirox use. Nephrology (Carlton). 2015;20(12):931-935.

98. Naderi M, Sadeghi-Bojd S, Valeshabad AK, et al. A prospective study of tubular dysfunction in pediatric patients with $\beta$-thalassemia major receiving deferasirox. Pediatr Hematol Oncol. 2013;30(8):748-754.

99. Teawtrakul N, Chansung K, Sirijerachai C, Wanitpongpun C, Thepsuthammarat K. The impact and disease burden of thalassemia in Thailand: a population-based study in 2010. J Med Assoc Thai. 2012; 95 Suppl 7:S211-S216.

100. Viprakasit V, Nuchprayoon I, Chuansumrit A, et al. Deferiprone(GPO${\mathrm{L}-O N E^{\circledR}}^{\circledR}$ monotherapy reduces iron overload in transfusion-dependent thalassemias: 1-year results from a multicenter prospective, single arm, open label, dose escalating phase III pediatric study (GPO-L-ONE; A001) from Thailand. Am J Hematol. 2013;88(4):251-260.

101. Kontoghiorghes GJ. Future chelation monotherapy and combination therapy strategies in thalassemia and other conditions: comparison of deferiprone, deferoxamine, ICL670, GT56-252, L1NAll and starch deferoxamine polymers. Hemoglobin. 2006;30(2):329-947.

102. Totadri S, Bansal D, Bhatia P, Attri SV, Trehan A, Marwaha RK. The deferiprone and deferasirox combination is efficacious in iron overloaded patients with $\beta$-thalassemia major: a prospective, single center, open-label study. Pediatr Blood Cancer. 2015;62(9): 1592-1596.

103. Cassinerio E, Orofino N, Roghi A, et al. Combination of deferasirox and deferoxamine in clinical practice: an alternative scheme of chelation in thalassemia major patients. Blood Cells Mol Dis. 2014; 53(3):164-167.

104. Elalfy MS, Adly AM, Wali Y, Tony S, Samir A, Elhenawy YI. Efficacy and safety of a novel combination of two oral chelators deferasirox/ deferiprone over deferoxamine/deferiprone in severely iron overloaded young beta thalassemia major patients. Eur J Haematol. 2015;95(5):411-420. 
105. Kolnagou A, Michaelides Y, Kontoghiorghe CN, Kontoghiorghes GJ The importance of spleen, spleen iron, and splenectomy for determining total body iron load, ferrikinetics, and iron toxicity in thalassemia major patients. Toxicol Mech Methods. 2013;23(1):34-41.

106. Kontoghiorghes GJ, Goddard JG, Bartlett AN, Sheppard L. Pharmacokinetic studies in humans with the oral iron chelator 1,2-dimethyl3-hydroxypyrid-4-one. Clin Pharmacol Ther. 1990;48(3):255-261.

107. Waldmeier F, Bruin GJ, Glaenzel U, et al. Pharmacokinetics, metabolism, and disposition of deferasirox in $\beta$-thalassemic patients with transfusion-dependent iron overload who are at pharmacokinetic steady state. Drug Metab Dispos. 2010;38(5):808-816.

108. Cao H. Pharmacological induction of fetal hemoglobin synthesis using histone deacetylase inhibitors. Hematology. 2004;9(3):223-233.

109. Ruchala P, Nemeth E. The pathophysiology and pharmacology of hepcidin. Trends Pharmacol Sci. 2014;35(3):155-161.

110. Arezes J, Nemeth E. Hepcidin and iron disorders: new biology and clinical approaches. Int J Lab Hematol. 2015;37 Suppl 1:92-98.

111. Kontoghiorghes GJ, Barr J, Nortey P, Sheppard L. Selection of a new generation of orally active $\alpha$-ketohydroxypyridine iron chelators intended for use in the treatment of iron overload. Am J Hematol. 1993;42(4):340-349.

112. Kontoghiorghe CN, Kolnagou A, Kontoghiorghes GJ. Potential clinical applications of chelating drugs in diseases targeting transferrin-bound iron and other metals. Expert Opin Investig Drugs. 2013;22(5):591-618.
113. Vreugdenhil G, Swaak AJ, de Jeu-Jaspers C, van Eijk HG. Correlation of iron exchange between the oral iron chelator 1,2-dimethyl-3hydroxypyrid-4-one (L1) and transferrin and possible antianaemic effects of L1 in rheumatoid arthritis. Ann Rheum Dis. 1990;49(11): 956-957.

114. Evans RW, Sharma M, Ogwang W, Patel KJ, Bartlett AN, Kontoghiorghes GJ. The effect of $\alpha$-ketohydroxypyridine chelators on transferrin saturation in vitro and in vivo. Drugs Today. 1992; 28 Suppl A:9-23.

115. Smeets ME, Vreugdenhil G, Holdrinet RS. Improvement of erythropoiesis during treatment with deferiprone in a patient with myelofibrosis and transfusional hemosiderosis. Am J Hematol. 1996; 51(3):243-244.

116. Chang YH, Shaw CF, Wu KH, Hsieh KH, Su YN, Lu PJ. Treatment with deferiprone for iron overload alleviates bone marrow failure in a Fanconi anemia patient. Hemoglobin. 2009;33(5):346-351.

117. Vreugdenhil G, Swaak AJ, Kontoghiorghes GJ, van Eijk HG. Efficacy and safety of oral iron chelator L1 in anaemic rheumatoid arthritis patients. Lancet. 1989;2(8676):1398-1399.

118. Mostert LJ, Van Dorst JA, Koster JF, Van Eijk HG, Kontoghiorghes GJ. Free radical and cytotoxic effects of chelators and their iron complexes in the hepatocyte. Free Radic Res Commun. 1987;3(6):379-388.

119. Brock JH, Licéaga J, Arthur HM, Kontoghiorghes GJ. Effect of novel 1-alkyl-3-hydroxy-2-methylpyrid-4-one chelators on uptake and release of iron from macrophages. Am J Hematol. 1990;34(1):21-25.

\section{Publish your work in this journal}

Drug Design, Development and Therapy is an international, peerreviewed open-access journal that spans the spectrum of drug design and development through to clinical applications. Clinical outcomes, patient safety, and programs for the development and effective, safe, and sustained use of medicines are a feature of the journal, which

\section{Dovepress}

has also been accepted for indexing on PubMed Central. The manuscript management system is completely online and includes a very quick and fair peer-review system, which is all easy to use. Visit http://www.dovepress.com/testimonials.php to read real quotes from published authors.

Submit your manuscript here: http://www.dovepress.com/drug-design-development-and-therapy-journal 